\title{
Directional dominance on stature and cognition in diverse human populations
}

\author{
A list of authors and their affiliations appears in the online version of the paper
}

Homozygosity has long been associated with rare, often devastating, Mendelian disorders ${ }^{1}$, and Darwin was one of the first to recognize that inbreeding reduces evolutionary fitness ${ }^{2}$. However, the effect of the more distant parental relatedness that is common in modern human populations is less well understood. Genomic data now allow us to investigate the effects of homozygosity on traits of public health importance by observing contiguous homozygous segments (runs of homozygosity), which are inferred to be homozygous along their complete length. Given the low levels of genome-wide homozygosity prevalent in most human populations, information is required on very large numbers of people to provide sufficient power $^{3,4}$. Here we use runs of homozygosity to study 16 healthrelated quantitative traits in 354,224 individuals from 102 cohorts, and find statistically significant associations between summed runs of homozygosity and four complex traits: height, forced expiratory lung volume in one second, general cognitive ability and educational attainment $\left(P<1 \times 10^{-300}, 2.1 \times 10^{-6}, 2.5 \times 10^{-10}\right.$ and $1.8 \times 10^{-10}$, respectively). In each case, increased homozygosity was associated with decreased trait value, equivalent to the offspring of first cousins being $1.2 \mathrm{~cm}$ shorter and having 10 months' less education. Similar effect sizes were found across four continental groups and populations with different degrees of genome-wide homozygosity, providing evidence that homozygosity, rather than confounding, directly contributes to phenotypic variance. Contrary to earlier reports in substantially smaller samples ${ }^{5,6}$, no evidence was seen of an influence of genome-wide homozygosity on blood pressure and low density lipoprotein cholesterol, or ten other cardio-metabolic traits. Since directional dominance is predicted for traits under directional evolutionary selection ${ }^{7}$, this study provides evidence that increased stature and cognitive function have been positively selected in human evolution, whereas many important risk factors for late-onset complex diseases may not have been.

Inbreeding influences complex traits through increases in homozygosity and corresponding reductions in heterozygosity, most likely resulting from the action of deleterious (partially) recessive mutations ${ }^{8}$. For polygenic traits, a systematic association with genome-wide homozygosity is not expected when dominant alleles at some loci increase the trait value while others decrease it. Rather, dominance must be biased in one direction on average over all causal loci, for instance to decrease the trait. Such directional dominance is expected to arise in evolutionary fitness-related traits due to directional selection $^{8}$. Studies of genome-wide homozygosity thus have the potential to reveal the non-additive allelic architecture of a trait and its evolutionary history. Historically, inbreeding has been measured using pedigrees $^{9}$. However, such techniques cannot account for the stochastic nature of inheritance, nor are they practical for the capture of the distant parental relatedness present in most modern-day populations. High-density genome-wide single nucleotide polymorphism (SNP) array data can now be used to assess genome-wide homozygosity directly, using genomic runs of homozygosity $(\mathrm{ROH})$. Such runs are inferred to be homozygous-by-descent and are common in human populations ${ }^{10,11}$. Summed $\mathrm{ROH}(\mathrm{SROH})$ is the sum of the length of these $\mathrm{ROH}$, in megabases of DNA. $F_{\mathrm{ROH}}$ is the ratio of SROH to the total length of the genome. Like pedigree-based $F$ (with which it is highly correlated $\left.{ }^{3}\right), F_{\mathrm{ROH}}$ estimates the probability of being homozygous at any site in the genome. $F_{\mathrm{ROH}}$ has been shown to vary widely within and between populations ${ }^{12}$ and is a powerful method of detecting genome-wide homozygosity effects ${ }^{13}$.

We found marked differences by geography and demographic history in both the population mean SROH and the relationship between $\mathrm{SROH}$ and $\mathrm{NROH}$ (the numbers of separate runs of homozygosity) (Fig. 1). As observed previously ${ }^{3,12,14}$, isolated populations have a higher burden of $\mathrm{ROH}$, whereas African heritage populations have the least homozygosity.

We studied $\beta_{F_{\mathrm{ROH}}}$, defined as the effect of $F_{\mathrm{ROH}}$ on 16 complex traits of biomedical importance (Fig. 2). For height, FEV1 (forced expiratory volume in one second, a measure of lung function), educational attainment, and $g$ (a measure of general cognitive ability derived from scores on several diverse cognitive tests), we found the effect sizes were greater than two intra-sex standard deviations, with $P$ values all less than $10^{-5}$. Thus the associations could not plausibly be explained by chance alone (Table 1; see Extended Data Figs 1-4 for Forest plots of individual traits; Supplementary Table 1 for s.d.). To ensure that the results were not driven by a few outliers, we repeated the analysis excluding extreme sub-cohort trait results. In all cases the effect sizes and their significance remained similar or increased (see Supplementary Table 2 for comparisons with and without outliers). After exclusion of outliers, these effect sizes translate into a reduction of $1.2 \mathrm{~cm}$ in height and $137 \mathrm{ml}$ in FEV1 for the offspring of first cousins, and into a decrease of 0.3 s.d. in $g$ and 10 months' less educational attainment.

We performed a number of analyses to exclude confounding. While $\mathrm{SROH}$ is wholly a genetic effect, its inheritance is entirely non-additive. Therefore, unlike in genome-wide association, an association with population genetic structure or co-segregation of additive genomewide polygenic effects and SROH (as opposed to SNPs in a genomewide association study) are not expected as a matter of course, except in the case of siblings. However, confounding could still theoretically arise, as discussed below. We therefore assessed this by conducting stratified and covariate analyses. We found effects of similar magnitude and in the same direction for all four traits across isolated and non-isolated European, Finnish, African, Hispanic, East Asian and South and Central Asian populations (Extended Data Fig. 5a and Supplementary Table 3). We further tested whether the effect sizes were similar when cohorts were split into more and less homozygous groups. The effect sizes were very similar, even though the degree of homozygosity (and variation in homozygosity) varied 3-10-fold between the two strata (depending on which cohorts contributed to the trait; Extended Data Fig. 5b). This suggests a broadly linear relationship with SROH. In general, confidence intervals overlap for stratified estimates, suggesting that differences arose due to sampling variance. Larger confidence intervals for some estimates reflect the lower power of some strata, in turn reflecting the sample size and degree of homozygosity of those strata (for example, the wider confidence intervals for estimates of educational attainment $\beta_{F_{\mathrm{ROH}}}$ for Finnish and African strata). Finally, we fitted educational attainment 


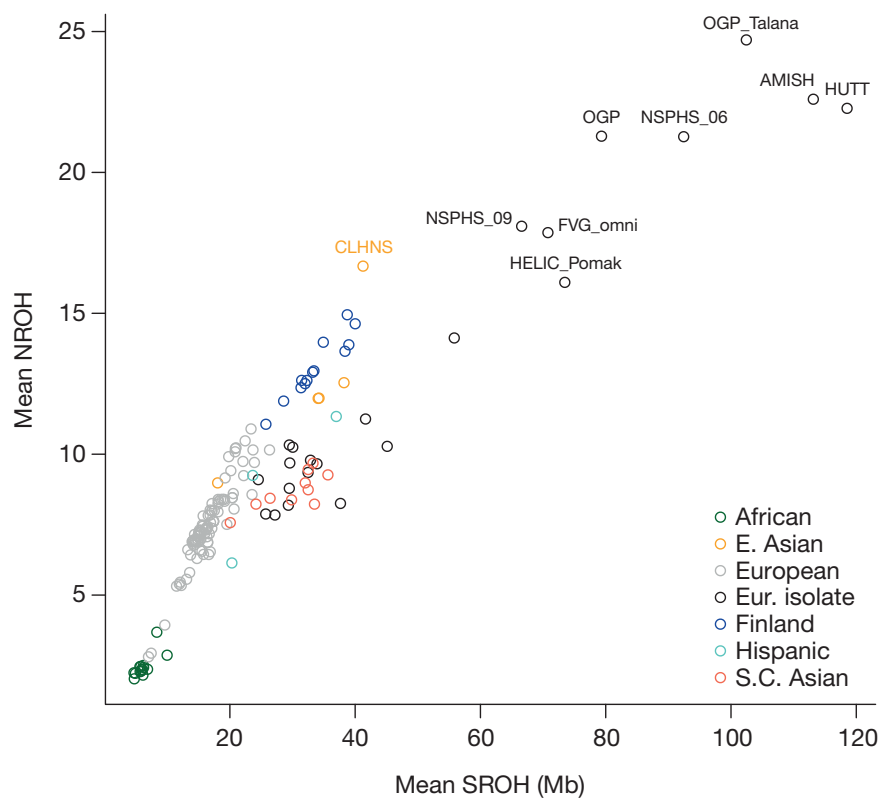

Figure $1 \mid$ Runs of homozygosity by cohort. The sum of runs of homozygosity (SROH) and the number of runs of homozygosity (NROH) are shown by sub-cohort. Populations differ by an order of magnitude in their mean burden of $\mathrm{ROH}$. There are clear differences by continent and population type both in the mean $\mathrm{SROH}$, and the relationship between $\mathrm{SROH}$ and $\mathrm{NROH}$. S.C. Asian, South and Central Asian; E. Asian, East Asian; Eur. Isolate, European isolates. The ten most homozygous cohorts are labelled: AMISH, Old Order Amish from Lancaster County, Pennsylvania; HUTT, Schmiedeleut Hutterites from South Dakota; NSPHS, northern Swedish population health study, 06 and 09 suffixes are different sampling years from different counties in northern Sweden; OGP, Ogliastra genetic park, Sardinia, Italy; Talana is a particular village in the region; FVG, Friuli-Venezia-Giulia genetic park, Italy, omni and 370 suffixes refer to subsets genotyped with the Illumina OmniX and $370 \mathrm{CNV}$ arrays; HELIC, Hellenic isolates, Greece, from Pomak villages in Thrace, and CLHNS, Cebu Longitudinal Health and Nutrition Survey in the Philippines.

as a proxy for potential confounding by socio-economic status; this covariate was available in sufficient (47) cohorts to maintain power. The estimated effect sizes for height, FEV1 and $g$ all reduced (17\%, 18\% and 35\%, respectively, Extended Data Fig. 5c), but this might have been expected given the known covariance between these three traits and educational attainment, and the association we found between educational attainment and $F_{\mathrm{ROH}}$. We found very small differences (3$11 \%$ reductions) in estimated $\beta_{F_{\mathrm{ROH}}}$ (Extended Data Fig. 6 and Supplementary Table 4), when comparing the fitting of polygenic mixed models as opposed to fixed-effect-only models, again suggesting that confounding (in this case due to polygenic effects arising from recent common ancestry) did not substantially affect the results.

Despite the observed 17-35\% reductions in estimated effect sizes for $F_{\mathrm{ROH}}$ on height, FEV1 and $g$, when fitting educational attainment as a covariate, the persistence of an effect suggests that most of the signals we observe are genetic. The consistency of effects with and without fitting relatedness and in particular in populations with very different degrees of homozygosity, all appear inconsistent with confounding as a result of environmental or additive genetic effects. As does the broad similarity in effect sizes across continents, although the relatively smaller numbers of cohorts of non-European descent meant we had limited power to detect intercontinental differences in effect sizes.

It is also interesting to consider the potential influence of assortative mating, which is commonly observed for human stature, cognition and education. The phenotypic extremes could be more genetically similar to each other and hence the offspring more homozygous, even if the highly polygenic trait architectures reduce this effect. However, at

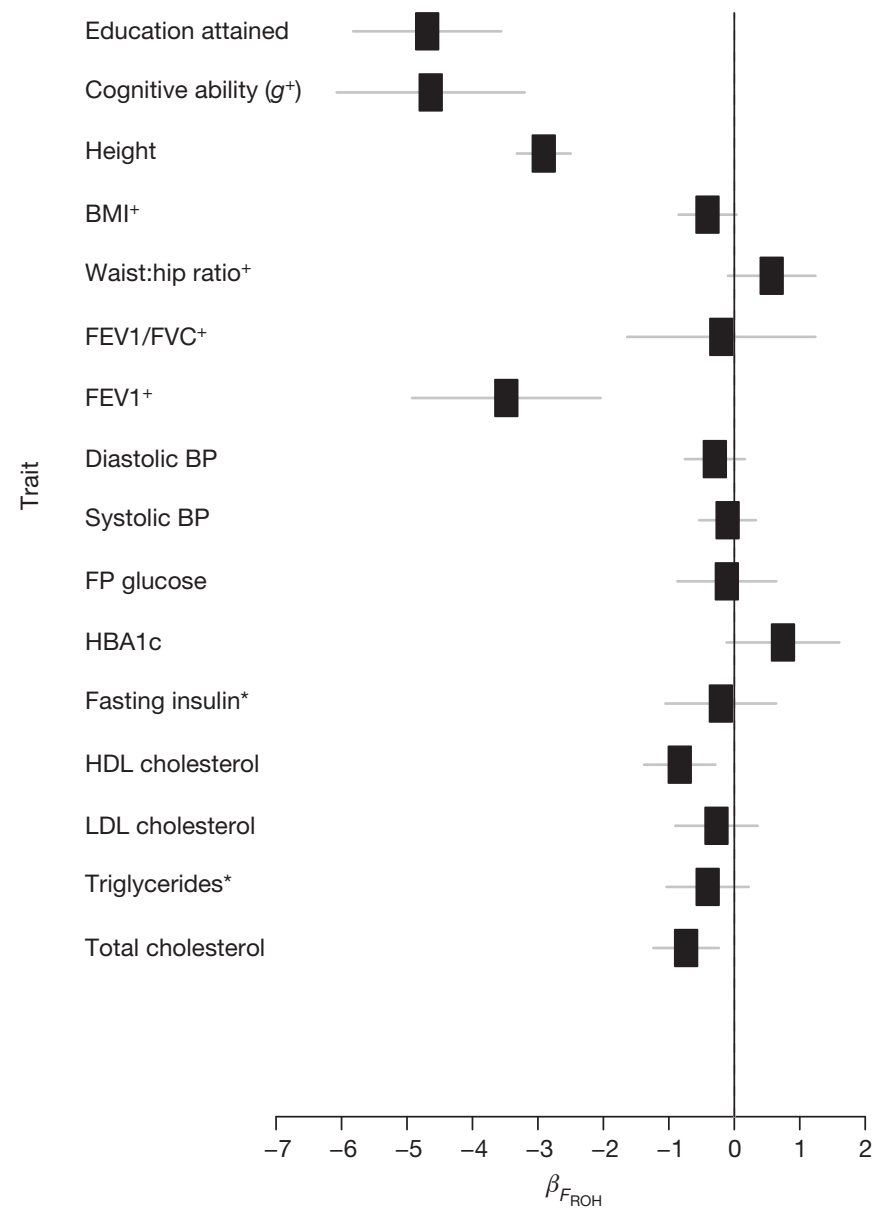

Figure $2 \mid$ Effects of genome-wide homozygosity, $\beta_{F_{\mathrm{ROH}}}$, on 16 traits. Four phenotypes show a significant effect of burden of ROH: height (145 subcohorts), FEV1 (34), educational attainment (47) and general cognitive ability, $g(23)$. HDL and total cholesterol are not significantly different from zero after correcting for 16 tests and no effect is observed for the other traits. To account for the different numbers of males and females in cohorts and marked effect of sex on some traits, trait units are intra-sex standard deviations. $\beta_{F_{\mathrm{ROH}}}$ is the estimated effect of $F_{\mathrm{ROH}}$ on the trait, where $F_{\mathrm{ROH}}$ is the ratio of the $\mathrm{SROH}$ to the total length of the genome. $95 \%$ confidence intervals are also plotted. Plus signs indicate that the phenotype was rank transformed, asterisks indicate that the phenotype was log transformed. $\mathrm{BMI}$, body mass index; $\mathrm{BP}$, blood pressure; FP fasting plasma; HbAlc, haemoglobin A1c (glycated haemoglobin); FEV1, forced expiratory volume in one second; FVC, forced vital capacity; HDL, high density lipoprotein; LDL, low density lipoprotein.

least in its simplest balanced form, the increase in genetic similarity would be equal at both ends of the phenotypic distribution, leading to no linear association between such genetic similarity and the trait; both tall and short people would be more homozygous. Furthermore, humans also mate assortatively on the basis of body mass index, for which we see no effect. A more complex possibility, a form of reverse causality, could arise when subjects from one trait extreme (for example, people with high educational attainment) are on average more geographically mobile, and thus have less homozygous offspring, with those offspring in turn inheriting the trait extreme concerned ${ }^{15}$. We do not think that this mechanism can account for our results, since it does not readily explain the constancy of our results under different models, especially the similarity in $\beta_{F_{\mathrm{ROH}}}$ for either more or less homozygous populations. Moreover, we observe similar effects in multiple single-village cohorts, and the Amish and Hutterites, where there is no geographic structure and/or no sampling of immigrants, hence such confounding by differential migration cannot occur. 
Table 1 | Effects of genome-wide burden of runs of homozygosity on four traits

\begin{tabular}{|c|c|c|c|c|c|}
\hline Phenotype & Outliers & Height & ${\text { FEV } 1^{+}}^{+}$ & Educational attainment & Cognitive ability (g) ${ }^{+}$ \\
\hline Subjects & & 354,224 & 64,446 & 84,725 & 53,300 \\
\hline$P$-association & Included & $<1 \times 10^{-300}$ & $2.1 \times 10^{-6}$ & $1.8 \times 10^{-10}$ & $2.5 \times 10^{-10}$ \\
\hline$P$-heterogeneity & Included & 0.014 & 0.10 & $1.2 \times 10^{-5}$ & 0.071 \\
\hline$\beta_{F_{R O H}}$-s.d. & Excluded & -2.91 & -3.48 & -4.69 & -4.64 \\
\hline s.e. $\beta_{F_{\text {POH }}}$-s.d. & Excluded & 0.21 & 0.73 & 0.58 & 0.73 \\
\hline$\beta_{F_{R O H}}$-units & Excluded & -0.188 & -2.2 & -12.9 & -4.64 \\
\hline s.e. $\beta_{F_{R O H}}$-units & Excluded & 0.014 & 0.46 & 1.83 & 0.73 \\
\hline Units & & $\mathrm{m}$ & litres & years & s.d. \\
\hline First cousin offspring effect & Excluded & -1.2 & -137 & -9.7 & -0.29 \\
\hline Units & & $\mathrm{cm}$ & $\mathrm{ml}$ & months & s.d. \\
\hline
\end{tabular}

$P$-association is the $P$ value for association; $P$-heterogeneity is the $P$ value for heterogeneity in a meta-analysis between trait and unpruned $F_{\mathrm{ROH}} ; \beta_{F_{\mathrm{ROH}}}$-s.d. is the effect size estimate of $F_{\mathrm{ROH}}$ expressed in units of intra-sex phenotypic standard deviations; $\beta_{F_{\mathrm{noO}}}$-units is the effect size estimate for $F_{\mathrm{ROH}}=1$ expressed in the measurement units; s.e., standard error. The $P$ values for those traits showing evidence for association are calculated, including five outlying cohort-specific effect size estimates (an outlier was defined as $t$-test statistic over 3 for the null hypothesis that the cohort effect size estimate equals the meta-analysis effect size estimate), which is conservative, as the majority of these are in the opposite direction. However, $\beta$ estimates exclude these outliers, for which there is evidence of discrepancy, and should thus be more accurate. A plus symbol indicates that the phenotype was rank transformed; FEV 1 is forced expiratory lung volume in one second; general cognitive ability is calculated as the first unrotated principal component of test scores across diverse domains of cognition.

Our estimate for the effect of homozygosity in height is consistent

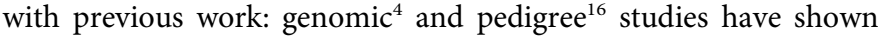
genome-wide homozygosity effects on stature with similar effect sizes (a 0.01 increase in $F$ decreases height by 0.037 s.d. ${ }^{16}$ versus 0.029 s.d. in the present study). We speculate that homozygosity is acting on a shared endophenotype of torso size which we detect in the height and FEV1 traits. The fact that the FEV1:FVC (forced vital capacity) ratio is not associated with $\mathrm{ROH}$ points to the effect acting on lung/chest size rather than airway calibre. The cognition effects cannot be wholly generated by height as an intermediate cause, given the greater proportion of variance explained for cognition, although we note that the correlation between height and cognition has been estimated as 0.16 (standard error, s.e. $=0.01$ ), and the genetic correlation (the correlation in additive genetic values) as 0.28 (s.e. $=0.09$; ref. 17 ).

Height is the canonical human complex trait, highly heritable and polygenic, with 697 genome-wide significant variants in 423 loci explaining $20 \%$ of the heritability and all common variants predicted to explain $60 \%$ of the heritability ${ }^{18}$. Most of the genetic architecture appears to be additive in nature, however $\mathrm{ROH}$ analysis reveals a distinct directional dominance component.

Our genomic confirmation of directional dominance for $g$ and discovery of genome-wide homozygosity effects on educational attainment in a wide range of human populations adds to our knowledge of the genetic underpinnings of cognitive differences, which are currently thought to be largely due to additive genetic effects ${ }^{19}$. Our findings go beyond earlier pedigree-based analyses of recent consanguinity to demonstrate that the observed effect of genome-wide homozygosity is not a result of confounding and influences demographically diverse populations across the globe. The estimated effect size is consistent with pedigree data (a 0.01 increase in $F$ decreases $g$ by 0.046 s.d. in our analysis and $0.029-0.048$ s.d. in pedigree-based studies $)^{20}$. It is germane to note that one extreme of cognitive function, early onset cognitive impairment, is strongly influenced by deleterious recessive loci ${ }^{21}$, so we can speculate that an accumulation of recessive variants of weaker effect may influence normal variation in cognitive function. Although increasing migration and panmixia have generated a secular trend in decreasing homozygosity ${ }^{22}$, the Flynn effect, wherein succeeding generations perform better on cognitive tests than their predecessors $^{23}$, this cannot be explained by our findings, because the intergenerational change in cognitive scores is much larger than the differences in homozygosity would predict. Likewise, the genomewide homozygosity effect on height cannot explain a significant proportion of the observed intergenerational increases ${ }^{24}$.

Inbreeding depression, which arises from the effect of genome-wide homozygosity, is ubiquitous in plants and is seen for numerous fitnessrelated traits in animals ${ }^{25}$, but we observed no effect for the 12 other mainly cardio-metabolic traits in which variation is strongly related to age. This suggests that previous reports in ecological studies or substantively smaller studies using pedigrees or relatively small numbers of genetic markers may have been false positives ${ }^{5,6}$. The lack of directional dominance on these traits does not, however, rule out a recessive component, as recessive variants acting in different directions will cancel out. Dominance variance is predicted to be greater for late-onset fitness traits ${ }^{26}$, so the lack of genome-wide homozygosity effects in the cardio-metabolic traits may be due to lack of directional dominance. $\mathrm{ROH}$ analyses within specific genomic regions are warranted to map recessive effects even when there is no genome-wide directional dominance. Such recessive effects have been observed for a subset of cardiovascular risk factors ${ }^{27}$ and expression traits ${ }^{28}$.

We have demonstrated the existence of directional dominance on four complex traits (stature, lung function, cognitive ability and educational attainment), while showing any effect on another 12 health-related traits is at least almost an order of magnitude smaller, non-linear or non-existent. This directional dominance implies that size and cognition (like schizophrenia protective alleles ${ }^{29}$ ) have been positively selected in human history - or at least that some variants increasing these traits contribute to fitness. However, the lack of any evidence for an association between many late-onset cardiovascular disease risk factors and $\mathrm{ROH}$ is perhaps surprising and suggests testing directly for an association between $\mathrm{ROH}$ and disease outcome. The magnitude of genome-wide homozygosity effects is relatively small in all cases, thus Darwin's supposition ${ }^{30}$ of "any evil [of inbreeding] being very small" is substantiated.

Online Content Methods, along with any additional Extended Data display items and Source Data, are available in the online version of the paper; references unique to these sections appear only in the online paper.

\section{Received 1 February; accepted 28 May 2015.}

Published online 1 July 2015.

1. Garrod, A. The incidence of alkaptonuria: a study of chemical individuality. Lancet 160, 1616-1620 (1902).

2. Darwin, C. The Variation of Animals and Plants Under Domestication (Appleton, 1868).

3. McQuillan, R. et al. Runs of homozygosity in European populations. Am. J. Hum. Genet. 83, 359-372 (2008).

4. McQuillan, R. et al. Evidence of inbreeding depression on human height. PLoS Genet. 8, e1002655 (2012).

5. Rudan, l. et al. Inbreeding and the genetic complexity of human hypertension. Genetics 163, 1011-1021 (2003).

6. Campbell, H. et al. Effects of genome-wide heterozygosity on a range of biomedically relevant human quantitative traits. Hum. Mol. Genet. 16, 233-241 (2007).

7. Charlesworth, D. \& Willis, J. H. The genetics of inbreeding depression. Nature Rev. Genet. 10, 783-796 (2009).

8. Wright, S. Evolution and the Genetics of Populations, Vol. 3: Experimental Results and Evolutionary Deductions (University of Chicago Press, 1977).

9. Wright, S. Coefficients of inbreeding and relationships. Am. Nat. 56, 330-339 (1922).

10. Broman, K. W. \& Weber, J. L. Long homozygous chromosomal segments in reference families from the Centre d'Étude du Polymorphisme Humain. Am. J. Hum. Genet. 65, 1493-1500 (1999). 
11. Gibson, J., Morton, N. E. \& Collins, A. Extended tracts of homozygosity in outbred human populations. Hum. Mol. Genet. 15, 789-795 (2006).

12. Kirin, M. et al. Genomic runs of homozygosity record population history and consanguinity. PLoS ONE 5, e13996 (2010).

13. Keller, M. C., Visscher, P. M. \& Goddard, M. E. Quantification of inbreeding due to distant ancestors and its detection using dense single nucleotide polymorphism data. Genetics 189, 237-249 (2011)

14. Pemberton, T. J. \& Rosenberg, N. A. Population-genetic influences on genomic estimates of the inbreeding coefficient: a global perspective. Hum. Hered. 77, 37-48 (2014)

15. Abdellaoui, A. et al. Educational attainment influences levels of homozygosity through migration and assortative mating. PLOS ONE 10, e0118935 (2015).

16. Neel, J. V. et al. The effects of parental consanguinity and inbreeding in Hirado, Japan. II. Physical development, tapping rate, blood pressure, intelligence quotient, and school performance. Am. J. Hum. Genet. 22, 263-286 (1970).

17. Marioni, R. E. et al. Common genetic variants explain the majority of the correlation between height and intelligence: the generation Scotland study. Behav. Genet. 44, 91-96 (2014).

18. Wood, A. R. et al. Defining the role of common variation in the genomic and biological architecture of adult human height. Nature Genet. 46, 1173-1186 (2014).

19. Deary, I. J. et al. Genetic contributions to stability and change in intelligence from childhood to old age. Nature 482, 212-215 (2012)

20. Morton, N. E. Effect of inbreeding on IQ and mental retardation. Proc. Natl Acad. Sci. USA 75, 3906-3908 (1978)

21. Najmabadi, H. et al. Deep sequencing reveals 50 novel genes for recessive cognitive disorders. Nature 478, 57-63 (2011).

22. Nalls, M. A. et al. Measures of autozygosity in decline: globalization, urbanization, and its implications for medical genetics. PLoS Genet. 5, e1000415 (2009).

23. Flynn, J. R. Massive IQ gains in 14 nations: what IQ tests really measure. Psychol. Bull. 101, 171-191 (1987).

24. Galton, F. Natural inheritance (MacMillan, 1889).

25. Hoffman, J. I. etal. High-throughput sequencing reveals inbreeding depression in a natural population. Proc. Natl Acad. Sci. USA 111, 3775-3780 (2014).

26. Wright, A., Charlesworth, B., Rudan, l., Carothers, A. \& Campbell, H. A polygenic basis for late-onset disease. Trends Genet. 19, 97-106 (2003).

27. Weiss, L. A., Pan, L., Abney, M. \& Ober, C. The sex-specific genetic architecture of quantitative traits in humans. Nature Genet. 38, 218-222 (2006).

28. Powell, J. E. et al. Congruence of additive and non-additive effects on gene expression estimated from pedigree and SNP data. PLoS Genet. 9, e1003502 (2013).

29. Keller, M. C. etal. Runs of homozygosity implicate autozygosity as a schizophrenia risk factor. PLoS Genet. 8, e1002656 (2012).

30. Darwin, C. The Effects of Crossing and Self Fertilization in the Vegetable Kingdom (John Murray, 1876).

Supplementary Information is available in the online version of the paper.

Acknowledgements This paper is the work of the ROHgen consortium. We thank the participants in all ROHgen studies; cohort-specific acknowledgements are detailed in Supplementary Table 6. This work was funded by a UK Medical Research Council (MRC) PhD studentship to P.K.J.; and J.F.W. and O.P. acknowledge support from the MRC Human Genetics Unit "QTL in Health and Disease" programme. We thank W. G. Hill for discussions and comments on the manuscript and K. Lindsay for administrative assistance.
Author contributions C.Hal, P.N.M.Me, H.B. N.J.S. D.C. D.A.M R.S.C. P.F. G.P. S.F.G H.H., L.F., R.A.S., A.D.M., C.N.P., G.De., P.D., L.B., U.L., S.I.B., C.M.L., N.J.T., A.Ton., P.B.M., T.I.S., C.N.R., D.K.A., A.J.O., S.L.K., B.B., G.Ga., A.P.M., J.G.E., M.J.W., N.G.M., S.C.H., J.M.S I.J.D., L.R.G., H.T., N.Pi., J.Ka., N.J.W., L.P., J.G.W., G.Gi., M.J.C., O.R., D.D.B., C.Gi., P.v.d.H., A.A.H., P.Kr., J.S., P.Kn., M.J., P.K.M., A.H., R.Sc., I.B.B., E.Va., D.M.B., D.B., K.L.M., M.B., C.M.v.D., D.K.S., A.Te., E.Z., A.Me., P.G., S.U., C.O., D.T., G.D.S., I.R., D.J.P., M.C., T.D.S., C.Hay, J.D., R.J.L., A.F.W., G.R.C., P.V., A.Sh., P.M.R., J.I.R., N.S., U.G., K.E.N., M.P., B.M.P. D.R.W., M.La., V.G., A.Ta., J.C.C., J.S.K., D.P.S., H.C., J.N.H., M.P., O.P. and J.F.W. designed individual studies. T.N., J.D.F., S.E., V.V., S.Tr., D.I.C., S.S.N., M.Ma., D.R., A.F., L.R.Y., E.H., C.Bo., J.R.P., S.C., U.B., G.M., T.Li., I.D., J.Z., J.P.B., E.S., S.Y., M.A.A., S.J.B., G.R.B., E.P.B., A.Ca., Y. Chan, S.J.C., Y.D.I.C., F.S.C., J.C., A.Co., L.Cu., G.Da., M.D., S.B.E., B.F., M.F.F., I.F., C.S.F., T.M.F., N.Fri., F.Ge., I.Gi., O.G., F.Gr., C.Gu., C.J.H., S.E.H., N.D.H., N.L.H., K.H., L.J.H. G.Ho., P.G.H., E.I., A.J., P.J., J.J., M.Ka., S.K., S.M.K., N.M.K., H.K.K., M.Ku., J.Ku., J.L., R.A.L., T.Le., D.C.L., L.Li., M.L.L., A.Lo., T.Lu., A.Lu., S.M., K.M., J.B.M., C.Mei., T.M., C.Men., F.D.M., L.M., G.W.M., R.H.M., R.N., M.N., M.S.N., G.T.O., A.O., S.P., W.R.P., J.S.P., I.Pa., K.P., N.Po., S.Ra., P.R., S.S.R., H.R., A.R., L.M.R., R.R., B.Sa., R.M.S., V.S., A.Sa., L.J.S., S.Se., P.S., B.H.S., N.Sor, A.V.St, M.G.S. K.S. N.Ta, K.D.T. B.O.T A.Tog M.To, J.T, A.G.U., A.v.H.V. T.V S.V E.VI., E.Vu., M.W., J.B.W., S.W., G.W., C.S.Y., G.Z., X.Z., M.Me., H.B., N.J.S., D.C., D.A.M., R.S.C., G.P., S.F.G., H.H., L.F., R.A.S., G.De., P.D., L.B., U.L., S.I.B., G.D.S., N.J.T., A.Ton., P.B.M., T.I.S., C.N.R. D.K.A. A.J.O, S.L.K B.B. M.K.K G.Ga, JG.E.M.J.W N.G.M S.C.H J.M.S, IJ.D, L.R.G., J.Ka., N.J.W., L.P., J.G.W., G.Gi., M.J.C., O.R., D.D.B., C.Gi., P.v.d.H., A.A.H., P.Kr., J.S., P.Kn., M.J., P.K.M., A.H., R.S., I.B.B., E.Va., D.M.B., D.B., K.L.M., M.B., C.M.v.D., D.K.S., E.Z. A.Me., P.G., C.O., D.T., D.J.P., M.C. T.D.S., C.Hay., R.J.L., A.F.W., G.R.C., P.V., A.Sh., P.M.R., J.I.R., N.S., U.G., M.P., B.M.P., D.R.W., M.La., J.C.C., J.S.K., D.P.S., J.N.H., M.P., O.P. and J.F.W. collected the data. S.Tr., D.I.C., M.C.C., C.Bo., U.B., I.D., M.A., F.W.A., S.J.B., D.J.B., E.B., E.P.B., A.Cc., S.J.C., J.C., I.F., T.M.F., C.Gu., C.J.H., T.B.H., N.D.H., M.I., E.I., J.J., P.Ko., M.Ku., L.J.L., R.A.L., L.Li., R.A.M., K.M., J.B.M., G.W.M., R.H.M., P.A.P., K.P., S.S.R., R.R., H.S., P.S. B.H.S., N.Sor., N.Sot., D.Va., J.B.W., C.S.Y., M.Me., N.J.S., D.C., D.A.M., R.S.C., P.F., G.P., S.F.G., H.H., L.F., G.De., P.D., L.B., U.L., S.I.B., C.M.L., A.Ton., P.B.M..,C.N.R., D.K.A., A.J.O., S.L.K., B.B., G.Ga., A.P.M., M.J.W., N.G.M., S.C.H., J.M.S., I.J.D., L.R.G., J.Ka., N.J.W., L.P. M.J.C., D.D.B., P.v.d.H., P.Kr., M.J., P.K.M., A.H., R.Sc., I.B.B., D.M.B., D.B., K.L.M., M.B., C.M.v.D., D.K.S., E.Z., A.Me., P.G., S.U., C.O., I.R., D.J.P., M.C., T.D.S., C.Hay., A.F.W., G.R.C., P.V., A.Sh., P.M.R., J.I.R., N.S., U.G., K.E.N., B.M.P., D.R.W., M.La., V.G., D.P.S., H.C., O.P. and J.F.W. contributed to funding. P.K.J., T.E., H.Ma., N.E., I.Ga., T.N., A.U.J., C.Sc.., A.V.Sm., W.Zhan., Y.O., A.Stc., J.D.F., W. Zhao, T.M.B., M.P.C., N.Fra., S.E., V.V., S.Tr., X.G., D.I.C., J.R.O., T.C., S.S.N.,Y.Chen, M.Ma., D.R., M.Ta., A.F., T.Kac., A.Bj., A.v.d.S., Y.W., A.K.G., L.R.Y., L.W., E.H., C.A.R., O.M., M.C.C., C.P., N.V., C.Ba., A.A.A., H.R.W., D.Vu., H.Me., J.R.P., S.S.Mi., M.C.B., S.S.Me., P.A.L., G.M., A.D., L.Y., L.F.B., D.Z., P.J.v.d.M., D.S., R.M., G.He., T.Kar., Z.W., T.Li., I.D., J.Z., W.M., L.La., S.W.v.L., J.P.B., A.R.W., A.Bo., T.S.A., L.M.H., E.S., S.Y., I.M.M., L.Ca H.G.d.H., M.A., U.A., N.A., F.W.A., S.E.B., S.B., A.Ca., Y. Chan, C.C., G.Da., G.E., B.F., M.F.F. F.Ge., M.G., S.E.H., J.J.H., J.H., J.E.H., P.G.H., A.J., Y.K., S.K., R.A.L., B.L., M.Lo., S.J.Loo., Y.L., P.M., A.Ma., C.Men., F.D.M., E.M., M.E.M., A.Mo., A.O., I.Pa., F.P., I.Pr., L.M.R., B.Sa., R.M.S., R.Sa.,H.S., W.R.S., C.Sa., C.Ma., B.Se., S.Sh., S.J.Lon., J.A.S.,L.S., R.J.S., M.J.S., S.Ta., B.O.T., A.Tog., M.To., N.Ts., J.v.S., S.V., D.Vo., E.B.W., W.W., J.Y., G.Z., N.J.S., R.A.S., A.D.M., C.N.P., S.I.B., N.J.T., A.P.M., S.C.H., H.T., N.Pi., L.P., P.v.d.H., P.Kr., R.Sc., I.B.B., A.Te., C.O., M.C., J.D., J.I.R., N.S., K.E.N., A.Ta., J.C.C., J.S.K. and D.P.S. analysed the data. P.K.J., T.E., H.Ma., N.E., I.Ga., T.N., A.U.J., C.Sc., A.V.Sm., M.C.B. and D.P.S. performed beta-testing of scripts. P.K.J. and T.E. performed the meta-analysis. P.K.J., T.E., O.P. and J.F.W. wrote the manuscript. All authors approved the final manuscript.

Author Information Reprints and permissions information is available at www.nature.com/reprints. Readers are welcome to comment on the online version of the paper. The authors declare competing financial interests: details are available in the online version of the paper. Correspondence and requests for materials should be addressed to J.F.W. (jim.wilson@ed.ac.uk). 


\section{METHODS}

Outline. Our aim was to look for an association between a genetic effect (SROH) and 16 complex traits. Our approach followed best practice genome-wide association meta-analysis (GWAMA) protocols, where applicable, except we had only one genetic effect to test.

Cohorts were invited to join based on known previous participation in GWAMA and willingness to participate. 159 sub-cohorts were created from 102 population-based or case-control genetic studies, by separating different genotyping arrays, cases and controls or ethnic sub-groups to ensure each sub-cohort was homogeneous. Within each of the 159 sub-cohorts we measured the association between $\mathrm{SROH}$ and trait using the following model. Where a sub-cohort had been ascertained on the basis of a disease status associated with a particular trait, that sub-cohort was excluded from the corresponding trait analysis.

Phenotype was regressed on genetic effect and known relevant covariates within each cohort, under the model specified in equation (1). The estimated genetic effect of $\mathrm{SROH}$ was then meta-analysed using inverse variance meta-analysis.

$$
Y=\mu+b_{1} \mathrm{SROH}+b_{2} \text { age }+b_{3} \mathrm{sex}+b_{4} \mathrm{PC} 1+b_{5} \mathrm{PC} 2+b_{6} \mathrm{PC} 3+e
$$

Where $\mathrm{Y}$ is the vector of trait values, $\mu$ the intercept, $\mathrm{b} 1$ the effect of SROH and $\mathrm{b} 2-6$ the effect of covariates. PC1-PC3, the post quality control within-cohort principal components of the cohort's relationship matrix and e the residual. Relationship matrices were determined genomically by each cohort using genome-wide array data. In addition, any other cohort-specific covariates known to be associated with the trait, including further principal components, and any trait-specific covariates and stratifications, such as medication and smoking status, were fitted as specified below. SROH was the sum of $\mathrm{ROH}$ called, with a length of at least $1.5 \mathrm{Mb}$ using PLINK31.

As is routine in GWAMA, for family-based studies only, we also fitted an additional term to account for additive genetic values and relatedness, using grammar+ type residuals and full hierarchical mixed modelling using $\mathrm{GenABEL}^{32}$ and $\mathrm{hglm}^{33}$, as specified in equation (2).

$$
Y=\mu+b_{1} \mathrm{SROH}+b_{2} \text { age }+b_{3} \mathrm{sex}+b_{4} \mathrm{PC} 1+b_{5} \mathrm{PC} 2+b_{6} \mathrm{PC} 3+Z a
$$

Where $a$ is the additive genetic value of each individual. $\operatorname{Var}(a)$ is assumed to be proportionate to the genomic relationship matrix (GRM) (a pedigree relationship matrix was used in the Framingham Heart Study). $Z$ is the identity matrix.

We then meta-analysed the regression coefficients $\left(b_{1}\right)$ of traits on SROH for the 159 sub-cohorts.

Cohort recruitment. Data from 102 independent genetic epidemiology studies of adults were included. All subjects gave written informed consent and studies were approved by the relevant research ethics committees. Homogeneous sub-cohorts were created for analysis on the basis of ethnicity, genotyping array or other factors. Where a cohort had multiple ethnicities, sub-cohorts for each separate ethnicity were created and analysed separately. In all cases individuals of European, African, South or Central Asian, East Asian and Hispanic heritage individuals were separated. In some cases sub-categories, such as Ashkenazi Jews, were also distinguished. Ethnic outliers were excluded, as were the second of any monozygotic twins and pregnant subjects. Continental ancestry of cohorts participating in each trait study is presented in Extended Data Table 1. Cohort genotyping and summary information are shown in Supplementary Table 6, with age, sex, trait and homozygosity summary statistics given in Supplementary Tables 9,10 and 11 . For case-control and trait-extreme studies, patients or extreme-only sub-cohorts were analysed separately to controls. Where case status was associated with the trait under analysis the sub-cohort was excluded from that study (see below).

Subjects within a sub-cohort were genotyped using the same SNP array, or where two very similar arrays were used (for example, Illumina OmniExpress and IlluminaOmni1), the intersection of SNPs on both arrays, provided the intersection exceeded 250,000 SNPs. Where a study used two different genotyping arrays, separate sub-cohorts were created for each array, and analysis was done separately. Paediatric cohorts were not included.

Genotyping. All subjects were genotyped using high-density genome-wide ( $>250,000$ SNP) arrays, from Illumina, Affymetrix or Perlegen. Custom arrays were not included. Each study's usual array-specific genotype quality control standards for genome-wide association were used and are shown in Supplementary Table 6. Only autosomal data were analysed.

Phenotyping. We studied 16 quantitative traits which are widely available and represent different domains related to health, morbidity and mortality: height, body mass index (BMI), waist:hip ratio (WHR), diastolic and systolic blood pressure (DBP, SBP), fasting plasma glucose (FPG), fasting insulin (FI), haemoglobin Alc (HbAlc), total cholesterol, HDL and LDL cholesterol levels, triglycerides, forced expiratory volume in one second (FEV1), ratio of FEV1 to forced vital capacity (FVC), general cognitive ability $(g$ ) and years of educational attainment Phenotypic quality control was performed locally to assess the accuracy and distribution of phenotypes and covariates. Further covariates were included when the relevant genome-wide association study consortium also included them. The trait categories were anthropometry, blood pressure, glycaemic traits, classical lipids, lung function, cognitive function and educational attainment, following models in the GIANT ${ }^{34}$, ICBP ${ }^{35}$, MAGIC $^{36}$, CHARGE $^{37}$, Spirometa ${ }^{38}$ and SSGAC $^{39}$ consortia. The model for FEV1 did not include height as a covariate Effect sizes for FEV1 therefore include size effects that also underpin height Studies assembled files containing study traits and the following covariates: sex, age, first three principal components of ancestry, lipid-lowering medication, eversmoker status, anti-hypertensive medication, diabetes status and year of birth Educational attainment was defined in accordance with the ISCED 1997 classification (UNESCO), leading to seven categories of educational attainment that are internationally comparable ${ }^{39}$. LDL values estimated using Friedewald's equation were accepted. Cohorts without fasting samples did not participate in the LDL-cholesterol, triglycerides, fasting insulin or fasting plasma glucose analyses. Cohorts with semi-fasting samples fitted a categorical or quantitative fasting time variable as a covariate. Subjects with less than $4 \mathrm{~h}$ fasting were not included.

Where subjects were ascertained, for example, on the basis of hypertension, that sub-cohort was excluded from analysis of traits associated with the disorder, for example blood pressure. The traits excluded from meta-analysis are as follows: ascertainment on type 2 diabetes, thus fasting insulin, $\mathrm{HbAlc}$ and fasting plasma glucose excluded; ascertainment on hypertension, thus blood pressures excluded; ascertainment on venous thrombosis or coronary artery disease, thus blood lipids excluded; ascertainment on obesity or the metabolic syndrome, thus blood lipids, body mass index, waist-hip ratio, fasting insulin and fasting plasma glucose excluded.

Somewhat unusually for a large consortium meta-analysis, the majority of the analysis after initial genotype and phenotype quality control was performed by a pipeline of standardised $\mathrm{R}$ and shell scripts, to ensure uniformity and reduce the risk of errors and ambiguities (available at https://www.wiki.ed.ac.uk/display/ $\mathrm{ROHgen} /$ Analysis + Plan + production + release +3.0 ). The pipeline was used for all stages from this point onwards.

Calling runs of homozygosity. SNPs with more than $3 \%$ missingness across individuals or with a minor allele frequency less than $5 \%$ were removed. $\mathrm{ROH}$ were defined as runs of at least 50 consecutive homozygous SNPs spanning at least $1,500 \mathrm{~kb}$, with less than a $1,000 \mathrm{~kb}$ gap between adjacent $\mathrm{ROH}$ and a density of SNP coverage within the $\mathrm{ROH}$ of no more than $50 \mathrm{~kb} / \mathrm{SNP}$, with one heterozygote and five no calls allowed per window, and were called using PLINK ${ }^{31}$, with the following settings: homozyg-window-snp 50; homozyg-snp 50; homozyg-kb 1500 ; homozyg-gap 1000; homozyg-density 50; homozyg-window-missing 5; homozyg-window-het 1 . The same criteria were used by McQuillan et al. ${ }^{3}$, except SNP density has been relaxed to avoid regions of sparser coverage (still including 50 SNPs) being missed. The sum of runs of homozygosity (SROH) was then calculated. $F_{\mathrm{ROH}}$ was calculated as $\mathrm{SROH} /\left(3 \times 10^{9}\right)$ reflecting the length of the autosomal genome. Copy number variants $(\mathrm{CNV})$ are known to influence cognition ${ }^{40}$; however, prior calling of $\mathrm{CNV}$ and $\mathrm{ROH}$ in one of our cohorts reduced the SROH by only $0.3 \%{ }^{3}$, making it implausible that deletions called as ROH influence our findings.

ROH called from different genotyping arrays. We show that SROH called with these parameters is relatively insensitive to the density and type of array used (Extended Data Fig. 7). We used 2.5 million SNPs available for 851 HapMap and 1000 Genomes Project ${ }^{41}$ samples from multiple continents to investigate the effect of array when using our ROH-calling parameters in PLINK. The data set included samples of African, European, admixed American, South and Eas Asian heritage. By subsampling SNPs from the 2.5 million we created array data for the commonly used Illumina CNV370 and OmniExpress beadchips and the Affymetrix6 array for each individual (see Supplementary Table 7 for details of the SNP numbers). The correlation in SROH using different arrays on the same individuals was $0.93-0.94$ for all pairwise chip comparisons.

Trait association with SROH. The association between trait and SROH was calculated using a linear model in accordance with equation (1). Additional covariates were fitted for some analyses (shown below) or for some cohorts where analysts were aware of study specific effects (for example, study centre). For BMI, WHR, FEV1, FEV1/FVC and $g$, trait residuals were calculated for the model excluding $\mathrm{SROH}$, these residuals were then rank-normalized and the effect of $\mathrm{SROH}$ on these rank-normalized residuals estimated. Triglycerides and fasting insulin were $\ln$-transformed. Additional covariates were as follows: age $^{2}$ was included as a covariate for all traits apart from height and $g$. BMI was included as a covariate for WHR, SBP, DBP, FPG, FI and HbAlc. Year of birth was included as a covariate for educational attainment and ever-smoking for FEV1 and FEV1/ FVC. Where a subject was known to be taking lipid-lowering medication, total 
cholesterol was adjusted by dividing by 0.8 . Similarly, where a subject was known to be taking anti-hypertensive medication, SBP and DBP measurements were increased by 15 and $10 \mathrm{~mm} \mathrm{Hg}$, respectively.

Where the cohort was known to have significant kinship, genetic relatedness was also fitted, using the mixed model, in accordance with equation (2). The polygenic model was fitted in GenABEL using the fixed covariates and the genomic relationship matrix ${ }^{32}$. GRAMMAR $+(\mathrm{GR}+)($ ref. 42$)$ residuals were then fitted to $\mathrm{SROH}$ as well as the full mixed model being fitted simultaneously, using GenABEL's hierarchical generalized linear model (HGLM) function ${ }^{33}$. Populations with kinship thus potentially had three estimates of $\beta_{F_{\mathrm{ROH}}}$ : using fixed effects only, and using the mixed model approaches, (GR+ and HGLM) for $\mathrm{SROH}$.

To investigate potential confounding, where available, educational attainment was added as an ordinal covariate and all models rerun, giving revised estimates of $\beta_{F_{\mathrm{ROH}}}$. This is potentially an over adjustment for $g$ due to the phenotypic and genetic correlations with educational attainment ${ }^{43}$. However it must be recognized that educational attainment does not capture all potential environmental confounding.

Cohort phenotypic means and standard deviations were checked visually for inter-cohort consistency, with apparent outliers then being corrected (for example, due to units or incorrectly specified missing values), explained (for example, due to different population characteristics) or excluded. Individual sub-cohort trait means and standard deviations are tabulated in Supplementary Table 9 and age and gender information is in Supplementary Table 10.

Meta-analysis. As is routine in genome-wide association meta-analyses, analysis was performed within homogeneous sub-populations and only meta-analysis of the estimated (within-population) effect sizes was used to combine results between populations, avoiding any confounding effects of inter-population differences in trait or genetic effect distributions. Inverse-variance meta-analysis of all subcohorts' effect estimates was performed using Rmeta, on a fixed-effect basis (Supplementary Table 5 compares random effects meta-analysis). In the principal analyses, for cohorts with relatedness, HGLM estimates of $\beta_{F_{\mathrm{ROH}}}$ were preferred; however, where HGLM had failed to converge, results using GRAMMAR + were included. These results were combined with those for unrelated cohorts on a fixedmodel-only basis. Result outliers were defined as individual cohort by trait results, which failed the hypothesis, cohort $\left(\beta_{F_{\mathrm{ROH}}}\right)=$ pre-quality-control meta-analysis $\left(\beta_{F_{\mathrm{ROH}}}\right)$, with a $t$-test statistic $>3$. Analyses were performed with and without outliers for $\beta_{F_{\mathrm{ROH}}}$ in phenotypic units and in intra-sex phenotypic standard deviations (Supplementary Table 8 ). The principal results we present are for $F_{\mathrm{ROH}}$ with outliers included for the hypothesis tests (which turns out to be more conservative), but with outliers excluded when estimating $\beta_{F_{\mathrm{ROH}}}$ (ref. 44). Metaanalysis was performed using inverse variance meta-analysis in the $\mathrm{R}$ package Rmeta, with $\beta_{F_{\mathrm{ROH}}}$ taken as a fixed effect and alternatively as a random effect. The principal results are on a fixed-effects basis, with Supplementary Table 5 showing comparison with the random-effects analysis.

Meta-analyses were re-run for various subsets, according to geographic and demographic features of the cohorts. Cohorts were divided into more homozygous and less homozygous strata with the boundary being set so each within-stratum meta-analysis had equal statistical power.

Data reporting. Randomization and blind allocation were not applicable to this study.

31. Purcell, S. PLINK: a tool set for whole-genome association and population-based linkage analyses. Am. J. Hum. Genet. 81, 559-575 (2007).

32. Aulchenko, Y. S., Ripke, S., Isaacs, A. \& van Duijn, C. M. GenABEL: an R library for genome-wide association analysis. Bioinformatics 23, 1294-1296 (2007)

33. Ronnegard, L., Shen, X. \& Alam, M. hglm: a package for fitting hierarchical generalized linear models. $R$ Journal 2, 20-28 (2010).

34. Lango Allen, H. et al. Hundreds of variants clustered in genomic loci and biological pathways affect human height. Nature $\mathbf{4 6 7}, \mathbf{8 3 2 - 8 3 8 ( 2 0 1 0 )}$

35. Ehret, G. B. et al. Genetic variants in novel pathways influence blood pressure and cardiovascular disease risk. Nature 478, 103-109 (2011)

36. Scott, R. A. et al. Large-scale association analyses identify new loci influencing glycemic traits and provide insight into the underlying biological pathways. Nature Genet. 44, 991-1005 (2012).

37. Willer, C. J. etal. Discovery and refinement of loci associated with lipid levels. Nature Genet. 45, 1274-1283 (2013).

38. Soler Artigas, M. etal. Genome-wide association and large-scale follow up identifies 16 new loci influencing lung function. Nature Genet. 43, 1082-1090 (2011).

39. Rietveld, C. A. et al. GWAS of 126,559 individuals identified genetic variants associated with educational attainment. Science 340, 1467-1471 (2013).

40. Stefansson, $\mathrm{H}$. et al CNVs conferring risk of autism or schizophrenia affect cognition in controls. Nature 505, 361-366 (2014).

41. An integrated map of genetic variation from 1,092 human genomes. Nature 491, 56-65 (2012).

42. Aulchenko, Y. S., de Koning, D. J. \& Haley, C. Genomewide rapid association using mixed model and regression: a fast and simple method for genome-wide pedigree-based quantitative trait loci association analysis. Genetics 177, 577-585 (2007).

43. Marioni, R. E. et al. Molecular genetic contributions to socioeconomic status and intelligence. Intelligence 44, 26-32 (2014)

44. Hedges, L. V. \& Olkin, I. Statistical Methods for Meta-Analysis (Academic Press, New York, 1985). 
Peter K. Joshi ${ }^{1 *}$, Tonu Esko $2,3,4,5 *$, Hannele Mattsson ${ }^{6,7}$, Niina Eklund ${ }^{6}$, Ilaria Gandin ${ }^{8}$, Teresa Nutile ${ }^{9}$, Anne U. Jackson ${ }^{10}$, Claudia Schurmann ${ }^{11,12}$, Albert V. Smith ${ }^{13,14}$, $Z_{\text {Zao }}{ }^{21}$, Traci M. Bartz ${ }^{22}$, Maria Pina Concas ${ }^{23}$, Nora Franceschini ${ }^{24}$, Stefan Enroth $^{25}$, Veronique Vitart ${ }^{26}$, Stella Trompet ${ }^{27}$, Xiuqing Guo ${ }^{28,29}$, Daniel I. Chasman ${ }^{30}$, Jeffrey R. $0^{\prime} C^{\prime}$ nne $\left.\right|^{31}$, Tanguy Corre ${ }^{32,33}$, Suraj S. Nongmaithem ${ }^{34}$, Yuning Chen ${ }^{35}$, Massimo Mangino $^{36,37}$, Daniela Ruggiero ${ }^{9}$, Michela Traglia ${ }^{38}$, Aliki-Eleni Farmaki ${ }^{39}$, Tim Kacprowski ${ }^{40}$, Andrew Bjonnes ${ }^{41}$, Ashley van der Spek ${ }^{42}$, Ying Wu ${ }^{43}$, Anil K. Giri ${ }^{44}$, Lisa R. Yanek ${ }^{45}$, Lihua Wan ${ }^{46}$, Edith Hofer ${ }^{47,48}$, Cornelius A. Rietveld ${ }^{49}$, Olga McLeod ${ }^{50}$, Marilyn C. Cornelis ${ }^{51,52}$, Cristian Pattaro ${ }^{53}$, Niek Verweij ${ }^{54}$, Clemens

Baumbach ${ }^{55,56,57}$, Abdel Abdellaoui ${ }^{58}$, Helen R. Warren ${ }^{59,60}$, Dragana Vuckovic ${ }^{8}$, Hao Mei $^{61}$, Claude Bouchard ${ }^{62}$, John R. B. Perry ${ }^{63}$, Stefania Cappellani ${ }^{64}$, Saira S. Mirza ${ }^{42}$ Miles C. Benton ${ }^{65}$. Ulrich Broeckel ${ }^{66}$, Sarah E. Medland ${ }^{67}$, Penelope A. Lind ${ }^{67}$, Giovann Malerba $^{68}$, Alexander Drong ${ }^{69}$, Loic Yengo ${ }^{70}$, Lawrence F. Bielak ${ }^{21}$, Degui Zhi ${ }^{71}$, Peter J. van der Most ${ }^{72}$, Daniel Shriner ${ }^{73}$, Reedik Mägi ${ }^{2}$, Gibran Hemani ${ }^{74}$, Tugce Karaderi ${ }^{69}$ Zhaoming Wang 82 , Tian Liu 7,78 , Ilja Demuth Wazaros Lataniotis
Wood $^{85}$, Amelie Bonnefond
${ }^{70}$, Tarunveer S. Ahluwalia Salvi $^{90}$, Seyhan Yazar $^{91}$, Lisbeth Carstensen ${ }^{92}$, Hugoline G. de Haan ${ }^{93}$, Mark Abney ${ }^{94}$ Uzma Afzal ${ }^{15,16}$, Matthew A. Allison ${ }^{95}$, Najaf Amin ${ }^{42}$, Folkert W. Asselbergs ${ }^{96,97,98}$ Stephan J. L. Bakker ${ }^{99}$, R. Graham Barr ${ }^{100}$, Sebastian E. Baumeister ${ }^{101}$, Daniel J. Benjamin ${ }^{102,103}$, Sven Bergmann ${ }^{32,33}$, Eric Boerwinkle ${ }^{104}$, Erwin P. Bottinger ${ }^{11}$, Archie Campbell ${ }^{105}$, Aravinda Chakravarti ${ }^{106}$, Yingleong Chan ${ }^{3,4,5}$, Stephen J. Chanock ${ }^{75}$, Constance Chen ${ }^{107}$, Y. -D. Ida Chen ${ }^{28,29}$, Francis S. Collins ${ }^{108}$, John Connell ${ }^{109}$, Adolfo Correa ${ }^{61}$, L. Adrienne Cupples ${ }^{35,110}$, George Davey Smith ${ }^{74}$, Gail Davies ${ }^{111,112}$, Marcus Dörr $^{113}$, Georg Ehret ${ }^{106,114}$, Stephen B. Ellis ${ }^{11}$, Bjarke Feenstra ${ }^{2}{ }^{2}$, Mary F. Feitosa ${ }^{46}$, lan Ford ${ }^{115}$, Caroline S. Fox ${ }^{110,116}$, Timothy M. Frayling ${ }^{55}$, Nele Friedrich ${ }^{117}$, Frank Geller ${ }^{92}$, Generation Scotland ${ }^{105}$, Irina Gillham-Nasenya ${ }^{36}$, Omri Gottesman ${ }^{11}$, Misa Graff $^{24}$, Francine Grodstein ${ }^{52}$, Charles Gu ${ }^{118}$, Chris Haleve ${ }^{26,119}$, Christopher J. Hammond ${ }^{36}$, Sarah E. Harris ${ }^{105,112}$, Tamara B. Harris ${ }^{120}$, Nicholas D. Hastie ${ }^{26}$ Nancy L. Heard-Costa ${ }^{110,121}$, Kauko Heikkilä122, Lynne J. Hocking ${ }^{123}$, Georg Homuth ${ }^{40}$, Jouke-Jan Hottenga ${ }^{58}$, Jinyan Huang ${ }^{124}$, Jennifer E. Huffman ${ }^{26}$, Pirro G. Hysi ${ }^{36}$, M. Arfan Ikram $^{42,125}$, Erik Ingelsson ${ }^{69,126}$, Anni Joensuu ${ }^{6,7}$, Asa Johansson ${ }^{25,127}$, Pekka Jousilahti $1^{128}$, J. Wouter Jukema ${ }^{129}$, Mika Kähönen ${ }^{130}$, Yoichiro Kamatani ${ }^{18}$, Stavroula Kanoni ${ }^{22}$, Shona M. Kerr ${ }^{26}$, Nazir M. Khan ${ }^{44}$, Philipp Koellinger ${ }^{49}$, Heikki A. Koistinen $131,132,133$, Manraj K. Kooner ${ }^{16}$, Michiaki Kubo ${ }^{134}$, Johanna Kuusisto ${ }^{135}$, Jari Lahti ${ }^{136,137}$, Lenore J. Launer ${ }^{120}$, Rodney A. Lea ${ }^{65}$, Benjamin Lehne ${ }^{15}$, Terho' Lehtimäki $^{138}$, David C.M. Liewald ${ }^{112}$, Lars Lind ${ }^{139}$, Marie Loh ${ }^{15,233}$, Marja-Liisa Lokki $^{140}$, Stephanie J. London ${ }^{141}$, Stephanie J. Loomis ${ }^{142}$, Anu Loukola ${ }^{122}$, Yingchang Lu $^{11,12}$, Thomas Lumley ${ }^{143}$, Annamari Lundqvist ${ }^{144}$, Satu Männistö ${ }^{28}$. Pedro Marques-Vidal $^{145}$, Corrado Masciullo ${ }^{38}$, Angela Matchan ${ }^{146}$, Rasika A. Mathias ${ }^{45,147}$, Koichi Matsuda ${ }^{148}$, James B. Meigs ${ }^{149}$, Christa Meisinger ${ }^{56}$, Thomas Meitinger ${ }^{150,151}$ Cristina Menni ${ }^{36}$, Frank D. Mentch ${ }^{84}$, Evelin Mihailov' ${ }^{2}$, Lili Milani ${ }^{2}$, May E. Montasser ${ }^{31}$ Grant W. Montgomery ${ }^{152}$, Alanna Morrison ${ }^{104}$, Richard H. Myers ${ }^{153}$, Rajiv Nadukuru ${ }^{11}$ Pau Navarro 26 , Mari Nelis ${ }^{2}$, Markku S. Nieminen ${ }^{154}$, Ilja M. Nolte ${ }^{72}$, George T. $0^{\prime}$ Connor ${ }^{110,155}$, Adesola Ogunniyi ${ }^{156}$, Sandosh Padmanabhan ${ }^{157}$, Walter R. Palmas ${ }^{100}$, James S. Pankow ${ }^{158}$, Inga Patarcic ${ }^{159}$, Francesca Pavani ${ }^{53}$, Patricia A. Peyser $^{21}$, Kirsi Pietilainen ${ }^{7,132,160}$, Neil Poulter ${ }^{161}$, Inga Prokopenko 162 , Sarju Ralhan ${ }^{163}$, Paul Redmond ${ }^{111}$, Stephen S. Rich ${ }^{164}$, Harri Rissanen ${ }^{144}$, Antonietta Robino ${ }^{64}$, Lynda M. Rose ${ }^{30}$, Richard Rose ${ }^{165}$, Cinzia Sala ${ }^{38}$, Babatunde Salako ${ }^{156}$, Veikko Salomaa ${ }^{128}$, Antti-Pekka Sarin ${ }^{6,7}$, Richa Saxena ${ }^{41}$, Helena Schmidt ${ }^{166}$, Laura J. Scott ${ }^{10}$, William R. Scott ${ }^{15,16}$, Bengt Sennblad ${ }^{50,167}$, Sudha Seshadri ${ }^{10,121}$, Peter Sever ${ }^{161}$ Smeeta Shrestha ${ }^{34}$, Blair H. Smith ${ }^{168}$, Jennifer A. Smith ${ }^{21}$, Nicole Soranzo ${ }^{146}$, Nona Sotoodehnia ${ }^{169}$, Lorraine Southam ${ }^{69,146}$, Alice V. Stanton ${ }^{170}$, Maria G. Stathopoulou ${ }^{171}$, Konstantin Strauch ${ }^{57,172}$, Rona J. Strawbridge ${ }^{50}$, Matthew J. Suderman ${ }^{74}$, Nikhil Tandon ${ }^{173}$, Sian-Tsun Tang ${ }^{174}$, Kent D. Taylor ${ }^{28,29}$, Bamidele $O$. Tayo ${ }^{175}$, Anna Maria Töglhofer 166, Maciej Tomaszewski ${ }^{89,176}$, Natalia Tšernikova 2,177 , Jaakko Tuomilehto ${ }^{131,188,179}$, Andre G. Uitterlinden ${ }^{42,180}$, Dhananjay Vaidya ${ }^{45,181}$ Astrid van Hylckama Vlieg ${ }^{93}$, Jessica van Setten ${ }^{83}$, Tuula Vasankari ${ }^{182}$, Sailaja Vedantam $^{3,4,5}$, Efthymia Vlachopoulou ${ }^{140}$, Diego Vozzi ${ }^{64}$, Eero Vuoksimaa ${ }^{122}$, Melanie Waldenberger ${ }^{55,56}$, Erin B. Ware ${ }^{21}$, William Wentworth-Shields ${ }^{94}$, John B. Whitfield ${ }^{183}$ Sarah Wild ${ }^{234}$, Gonneke Willemsen ${ }^{58}$, Chittaranjan S. Yajnik ${ }^{184}$, Jie Yao ${ }^{28}$, Gianluigi Zaza $^{185}$, Xiaofeng Zhu ${ }^{186}$, The BioBank Japan Project ${ }^{18}$, Rany M. Salem ${ }^{3,4,5}$, Mads Melbye $^{92,187}$, Hans Bisgaard ${ }^{86}$, Nilesh J. Samani ${ }^{89,176}$, Daniele Cusi ${ }^{90}$, David A. Mackey ${ }^{91}$, Richard S. Cooper ${ }^{175}$, Philippe Froguel ${ }^{70,162}$, Gerard Pasterkamp ${ }^{83}$, Struan F.A. Grant ${ }^{44,188}$, Hakon Hakonarson ${ }^{84,188}$, Luigi Ferrucci ${ }^{189}$, Robert A. Scott ${ }^{63}$, Andrew D. Morris ${ }^{190}$, Colin N. A. Palmer ${ }^{191}$, George Dedoussis ${ }^{39}$, Panos Deloukas ${ }^{82,192}$, Lars Bertram $^{78,193,235}$, Ulman Lindenberger ${ }^{79}$, Sonja I. Berndt ${ }^{7}$, Cecilia M. Lindgren ${ }^{4,69}$, Nicholas J. Timpson ${ }^{74}$, Anke Tönjes ${ }^{194}$, Patricia B. Munroe ${ }^{59,60}$, Thorkild I. A. Sørensen ${ }^{74,88,195}$, Charles N. Rotimi ${ }^{73}$, Donna K. Arnett ${ }^{196}$, Albertine J. Oldehinkel ${ }^{197}$, Sharon L. R. Kardia ${ }^{21}$, Beverley Balkau ${ }^{198}$, Giovanni Gambaro ${ }^{199}$, Andrew P. Morris $^{2,69,200}$, Johan G. Eriksson ${ }^{128,201,202,203,204}$, Margie J. Wright ${ }^{205}$, Nicholas G. Martin ${ }^{183}$, Steven C. Hunt ${ }^{206}$, John M. Starr ${ }^{112,207}$, Ian J. Deary ${ }^{111,112}$, Lyn R. Griffiths ${ }^{65}$ Henning Tiemeier ${ }^{42,208}$, Nicola Pirastu, 8,64 , Jaakko Kaprio, 122,209, Nicholas J. Wareham $^{63}$, Louis Pérusse ${ }^{210}$, James G. Wilson ${ }^{211}$, Giorgia Girotto ${ }^{8}$. Mark J. Caulfield $^{59,60}$, Olli Raitakari 212,213 , Dorret I. Boomsma ${ }^{58}$, Christian Gieger ${ }^{55,56,57}$, Pim van der Harst ${ }^{44,97,214}$, Andrew A. Hicks ${ }^{53}$, Peter Kraft ${ }^{107}$, Juha Sinisalo ${ }^{154}$, Paul $K^{\prime}$ knt $^{144}$, Magnus Johannesson ${ }^{215}$, Patrik K. E. Magnusson ${ }^{216}$, Anders Hamsten ${ }^{50}$ Reinhold Schmidt ${ }^{77}$, Ingrid B. Borecki ${ }^{217}$, Erkki Vartiainen ${ }^{128}$, Diane M. Becker ${ }^{45,218}$ Dwaipayan Bharadwaj ${ }^{4,236}$, Karen L. Mohlke ${ }^{43}$, Michael Boehnke ${ }^{10}$, Cornelia M. van Duijn $^{42}$, Dharambir K. Sanghera ${ }^{219,220}$, Alexander Teumer ${ }^{101}$, Eleftheria Zeggini ${ }^{146}$ Andres Metspalu 2,177 , Paolo Gasparini ${ }^{64,221}$, Sheila Ulivi ${ }^{64}$, Carole Ober ${ }^{94}$, Daniela Toniolo $^{38}$, Igor Rudan ${ }^{1}$, David J. Porteous ${ }^{105,112}$, Marina Ciullo ${ }^{9}$, Tim D. Spector ${ }^{36}$ Caroline Hayward ${ }^{26}$, Josée Dupuis ${ }^{35,110}$, Ruth J. F. Loos ${ }^{11,12,222}$, Alan F. Wright ${ }^{26}$, Giriraj R. Chandak ${ }^{34,223}$, Peter Vollenweider ${ }^{145}$, Alan R. Shuldiner ${ }^{31,224,225}$, Paul M.
Ridker $^{30}$, Jerome I. Rotter 28,29 , Naveed Sattar ${ }^{226}$, Ulf Gyllensten ${ }^{25}$, Kari E. North 24,227, Mario Pirastu ${ }^{23}$, Bruce M. Psaty ${ }^{228,229}$, David R. Weir ${ }^{20}$, Markku Laakso ${ }^{135}$, Vilmundur Gudnason ${ }^{13,14}$, Atsushi Takahashi ${ }^{18}$, John C. Chambers ${ }^{15,16,230}$, Jaspal S. Kooner ${ }^{16,174,230}$ David P. Strachan ${ }^{231}$, Harry Campbell ${ }^{1}$, Joel N. Hirschhorn ${ }^{3,4,5}$ Markus Perola ${ }^{2,6}$, Ozren Polašek $^{1,159}$ *' \& James F. Wilson ${ }^{1,26 *}$

${ }^{1}$ Centre for Global Health Research, Usher Institute for Population Health Sciences and Informatics, University of Edinburgh, Teviot Place, Edinburgh EH8 9AG, UK. ${ }^{2}$ Estonian Genome Center, University of Tartu, Riia 23b, 51010, Tartu, Estonia. ${ }^{3}$ Division of Endocrinology and Center for Basic and Translational Obesity Research, Boston Children's Hospital, Cambridge, 02141 Massachusetts, USA. ${ }^{4}$ Program in Medical and Population Genetics, Broad Institute, Cambridge Center 7, Cambridge, Massachusetts 02242, USA. ${ }^{5}$ Department of Genetics, Harvard Medical School, 25 Shattuck St, Boston, Massachusetts 02115, USA. 'Unit of Public Health Genomics, National Institute for Health and Welfare, P.O. Box 104, Helsinki, FI-00251, Finland. ${ }^{7}$ Institute for Molecular Medicine Finland (FIMM), University of Helsinki, P.O. Box 20, Helsinki, FI-00014, Finland.

${ }^{8}$ Department of Medical Sciences, University of Trieste, Strada di Fiume 447 - Osp. di Cattinara, 34149 Trieste, Italy. Institute of Genetics and Biophysics "A. Buzzati-Traverso" CNR, via Pietro Castellino, 111, 80131 Naples, Italy. ${ }^{10}$ Department of Biostatistics and Center for Statistical Genetics, University of Michigan, Ann Arbor, Michigan 48109, USA.

${ }^{11}$ The Charles Bronfman Institute for Personalized Medicine, Icahn School of Medicine at Mount Sinai, One Gustave L. Levy Place, New York, New York 10029, USA. ${ }^{12}$ The Genetics of Obesity and Related Metabolic Traits Program, Icahn School of Medicine at Mount Sinai, One Gustave L. Levy Place, New York, New York 10029, USA. ${ }^{13}$ Icelandic Heart Association, Holtasmari 1, 201, Kopavogur, Iceland. ${ }^{14}$ Faculty of Medicine, University of Iceland, Reykjavik, 101 , Iceland. ${ }^{15}$ Department of Epidemiology and Biostatistics, Imperial College London, Norfolk Place, London W2 1PG, UK. ${ }^{16}$ Department of Cardiology, Ealing Hospital NHS Trust, Uxbridge Road, Southall, Middlesex UB1 3HW, UK. ${ }^{17}$ Department of Human Genetics and Disease Diversity, Graduate School of Medical and Dental Sciences, Tokyo Medical and Dental University, 1-5-45 Yushima, Bunkyo-ku, Tokyo, 113-8510, Japan. ${ }^{18}$ Laboratory for Statistical Analysis, RIKEN Center for Integrative Medical Sciences, 1-7-22 Suehiro-cho, Tsurumi-ku, Yokohama, Kanagawa, 230-0045, Japan. ${ }^{19}$ Department of Medicine, University of Eastern Finland, 70210 Kuopio, Finland. ${ }^{20}$ Institute for Social Research, University of Michigan, 426 Thompson Street, Ann Arbor, Michigan 48104, USA. ${ }^{21}$ Department of Epidemiology, University of Michigan, 1415 Washington Heights, Ann Arbor, Michigan 48109, USA. ${ }^{22}$ Cardiovascular Health Research Unit, Departments of Biostatistics and Medicine, University of Washington, 1730 Minor Ave, Suite 1360, Seattle, Washington 98101, USA. ${ }^{23}$ Institute of Population Genetics, National Research Council, Trav. La Crucca n. 3 - Reg. Baldinca, 07100 Sassari Italy. ${ }^{24}$ Epidemiology, University of North Carolina, 137 E. Franklin St., Suite 306, Chapel Hill, North Carolina 27599, USA. ${ }^{25}$ Department of Immunology, Genetics, and Pathology, Biomedical Center, SciLifeLab Uppsala, Uppsala University, SE-75108 Uppsala, Sweden. ${ }^{26} \mathrm{MRC}$ Human Genetics Unit, Institute of Genetics and Molecular Medicine, University of Edinburgh, Crewe Road, EH4 2XU Edinburgh, UK. ${ }^{27}$ Department of Gerontology and Geriatrics, Leiden University Medical Center, PO Box 9600, Leiden, 2300 RC, The Netherlands. ${ }^{28}$ Institute for Translational Genomics and Population Sciences, Los Angeles Biomedical Research Institute, 1124 W. Carson Street, Torrance, California 90502, USA ${ }^{29}$ Department of Pediatrics, Harbor-UCLA Medical Center, Torrance, California 90502, USA. ${ }^{30}$ Division of Preventive Medicine, Brigham and Women's Hospital, 900 Commonwealth Avenue, East, Harvard Medical School, Boston, Boston, Massachusetts 02215, USA. ${ }^{31}$ Division of Endocrinology, Diabetes, and Nutrition and Program for Personalised and Genomic Medicine, Department of Medicine, University of Maryland School of Medicine, 685 Baltimore St. MSTF, Baltimore, Maryland 21201, USA.

${ }^{32}$ Department of Medical Genetics, University of Lausanne, Rue du Bugnon 27, Lausanne, 1005 , Switzerland. ${ }^{3}$ Swiss Institute of Bioinformatics, Quartier Sorge - batiment génopode, Lausanne, 1015, Switzerland. ${ }^{34}$ Genomic Research on Complex Diseases (GRC) Group, CSIR-Centre for Cellular and Molecular Biology, Habshiguda, Uppal Road, Hyderabad, 500007, India. ${ }^{35}$ Department of Biostatistics, Boston University School of Public Health, 801 Massachusetts Avenue, Boston, Massachusetts 02118, USA. ${ }^{36}$ Department of Twin Research \& Genetic Epidemiology, King's College London, South Wing, Block D, 3rd Floor, Westminster Bridge Road, London SE1 7EH, UK. ${ }^{37} \mathrm{NIHR}$ Biomedical Research Centre, Guy's and St. Thomas' Foundation Trust, Westminster Bridge Road, London SE1 7EH, UK. ${ }^{38}$ Division of Genetics and Cell Biology, San Raffaele Scientific Institute, Via Olgettina 58, 20132 Milano, Italy ${ }^{39}$ Department of Nutrition and Dietetics, Harokopio University of Athens, 70, El. Venizelou Ave, Athens 17671, Greece. ${ }^{40}$ Interfaculty Institute for Genetics and Functional Genomics, University Medicine Greifswald, Friedrich-Ludwig-Jahn-Str. 15A, Greifswald 17475, Germany. ${ }^{41}$ Center for Human Genetic Research, 55 Fruit Street, Massachusetts General Hospital, Massachusetts 02114, USA. ${ }^{42}$ Department of Epidemiology, Erasmus Medical Center, PO Box 2040, Rotterdam, 3000 CA, The Netherlands. ${ }^{43}$ Department of Genetics, University of North Carolina, Chapel Hill, North Carolina 27599, USA. ${ }^{44}$ Genomics and Molecular Medicine, CSIR-Institute of Genomics \& Integrative Biology, Mathura Road, New Delhi, 110025, India. ${ }^{45}$ The GeneSTAR Research Program, Division of General Internal Medicine, Department of Medicine, The Johns Hopkins University School of Medicine, Baltimore, Maryland 21287, USA. ${ }^{46}$ Department of Genetics, Washington University School of Medicine, 4444 Forest Park Boulevard, Saint Louis, Missouri 63108, USA.

${ }^{47}$ Department of Neurology, Clinical Division of Neurogeriatrics, Medical University Graz, Auenbruggerplatz 22, Graz, A-8036, Austria. ${ }^{48}$ Institute for Medical Informatics, Statistics and Documentation, Medical University Graz, Auenbruggerplatz2, Graz, A-8036, Austria. ${ }^{49}$ Erasmus School of Economics, Erasmus University Rotterdam, Burgemeester Oudlaan 50, Rotterdam, 3000 DR, The Netherlands. ${ }^{50}$ Atherosclerosis Research Unit, Department of Medicine Solna, Karolinska Institutet, CMM L8:03, Karolinska University Hospital, Solna, Stockholm, 171 76, Sweden. ${ }^{51}$ Channing Division of Network Medicine, Brigham \& Women's Hospital, 181 Longwood, Boston, Massachusetts 02115, USA. ${ }^{52}$ Nutrition, Harvard School of Public Health, 401 Park Drive, Boston, Massachusetts 02215, USA

${ }^{53}$ Center for Biomedicine, European Academy Bozen/Bolzano (EURAC), 39100 Bolzano, 
Italy (affiliated Institute of the University of Lübeck, D-23562 Lübeck, Germany). ${ }^{54}$ University of Groningen, University Medical Center Groningen, Department of Cardiology, Hanzeplein 1, Groningen, 9700 RB, The Netherlands. ${ }^{55}$ Research Unit of Molecular Epidemiology, Helmholtz Zentrum München, German Research Center for Environmental Health, Ingolstädter Landstr. 1, Neuherberg 85764, Germany. ${ }^{56}$ Institute of Epidemiology II, Helmholtz Zentrum München, German Research Center for Environmental Health, Ingolstädter Landstr. 1, Neuherberg 85764, Germany. ${ }^{57}$ Institute of Genetic Epidemiology, Helmholtz Zentrum München, German Research Center for Environmental Health, Ingolstädter Landstr. 1, Neuherberg 85764, Germany. ${ }^{58}$ Department of Biological Psychology, VU University Amsterdam, Van der Boechorststraat 1, Amsterdam, 1081 BT, The Netherlands. ${ }^{59}$ Clinical Pharmacology, William Harvey Research Institute, Barts and The London School of Medicine and Dentistry, Queen Mary University of London, Charterhouse Square, London EC1M 6BQ, UK. ${ }^{60}$ NIHR Barts Cardiovascular Biomedical Research Unit, Queen Mary University of London, Charterhouse Square, London EC1M 6BQ, UK. ${ }^{61}$ Department of Medicine, University of Mississippi Medical Center, 2500 N. State St., Jackson, Mississippi 39216, USA. ${ }^{62}$ Pennington Biomedical Research Center, 6400 Perkins Rd, Baton Rouge, Louisiana 70808, USA. ${ }^{63}$ MRC Epidemiology Unit, University of Cambridge School of Clinical Medicine, Cambridge Biomedical Campus, Cambridge CB2 0QQ, UK. ${ }^{64}$ Institute for Maternal and Child Health - IRCCS "Burlo Garofolo", via dell'Istria 65, 34137 Trieste, Italy. ${ }^{65}$ Institute of Health and Biomedical Innovation, Queensland University of Technology, 60 Musk Avenue, Kelvin Grove, GPO Box 2434, Brisbane Queensland 4001 Australia. ${ }^{66}$ Department of Pediatrics, Medical College of Wisconsin, 8701 Watertown Plank Rd, Milwaukee, Wisconsin 53226, USA. ${ }^{67}$ Quantitative Genetics, QIMR Berghofer Medical Research Institute, 300 Herston Rd, Herston, Brisbane Queensland 4006, Australia. ${ }^{68}$ Dipartimento di Scienze della Vita e della Riproduzione, University of Verona, Strada Le Grazie 15, 37134 Verona, Italy. ${ }^{69}$ Wellcome Trust Centre for Human Genetics, University of Oxford, Roosevelt Drive, Oxford OX3 7BN, UK. ${ }^{70}$ CNRS UMR 8199, European Genomic Institute for Diabetes (EGID), Lille 2 University, 1 Rue du Professeur Calmette, 59000 Lille, France. ${ }^{71}$ Department of Biostatistics, University of Alabama at Birmingham, 1665 University Blvd, Birmingham, Alabama 35294, USA. ${ }^{72}$ Department of Epidemiology, University of Groningen, University Medical Center Groningen, Groningen P.O. box 30.001, 9700 RB, Groningen, The Netherlands. ${ }^{73}$ Center for Research on Genomics and Global Health, National Human Genome Research Institute, Building 12A Room 4047, 12 South Dr., Bethesda, Maryland 20892, USA. ${ }^{74}$ MRC Integrative Epidemiology Unit, University of Bristol, Oakfield House, Oakfield Grove, Bristol BS8 2BN UK. ${ }^{75}$ Division of Cancer Epidemiology and Genetics, National Cancer Institute, National Institutes of Health, 9609 Medical Center Drive, Rockville, Maryland 20850, USA ${ }^{76}$ Cancer Genomics Research Laboratory, National Cancer Institute, SAIC-Frederick, Inc. Frederick National Laboratory for Cancer Research, Frederick, Maryland 21702, USA.

${ }^{77}$ Center for Lifespan Psychology, Max Planck Institute for Human Development, Lentzeallee 94, Berlin 14195, Germany. ${ }^{78}$ Vertebrate Genomics, Max Planck Institute for Molecular Genetics, Ihnestr. 72, Berlin, 14195 Germany. ${ }^{79}$ Charité Research Group on Geriatrics, Charité - Universitätsmedizin Berlin, Reinickendorferstr. 61, 13347 Berlin, Germany. ${ }^{80}$ Institute of Medical and Human Genetics, Charité - Universitätsmedizin Berlin, Augustenburger Platz 1, Berlin 13353, Germany. ${ }^{81}$ Division of Population Health Sciences, Medical Research Institute, University of Dundee, Ninewells Hospital and Schoo of Medicine, Dundee DD2 4BF, UK. ${ }^{82}$ William Harvey Research Institute, Barts and The London School of Medicine and Dentistry, Queen Mary University of London,

Charterhouse Square, London, EC1M 6BQ, UK ${ }^{83}$ Experimental Cardiology, Division Hear and Lungs, University Medical Center Utrecht, Heidelberglaan 100, Utrecht, 3584 CX, The Netherlands. ${ }^{84}$ Center for Applied Genomics, Children's Hospital of Philadelphia, 3615 Civic Center Boulevard, Philadelphia, Pennsylvania 19104, USA. ${ }^{85}$ Genetics of Complex Traits, University of Exeter Medical School, University of Exeter, Royal Devon and Exeter Hospital, Barrack Road, Exeter EX2 5DW, UK. ${ }^{86}$ COPSAC, Copenhagen Prospective Studies on Asthma in Childhood, Herlev and Gentofte Hospital, University of Copenhagen, Ledreborg Allé 34, DK-2820 Copenhagen, Denmark. ${ }^{88}$ Novo Nordisk Centre for Basic Metabolic Research, Section of Metabolic Genetics, Faculty of Health and Medical Sciences, University of Copenhagen, Universitetsparken 1, Copenhagen, 2100, Denmark ${ }^{89}$ Department of Cardiovascular Sciences, University of Leicester, BHF Cardiovascula Research Centre, Glenfield Hospital, Groby Road, Leicester LE3 9QP, UK. ${ }^{90}$ Department of Health Sciences, University of Milan, via A. di Rudini 8, 20142 Milan, Italy. ${ }^{91}$ Centre for Ophthalmology and Visual Science, University of Western Australia, Lions Eye Institute, 2 Verdun Street, Perth, Western Australia 6009, Australia. ${ }^{92}$ Department of Epidemiology Research, Statens Serum Institut, Artillerivej 5, Copenhagen, 2300, Denmark. ${ }^{93}$ Clinical Epidemiology, Leiden University Medical Center, PO Box 9600, Leiden, 2300 RC, The Netherlands. ${ }^{94}$ Department of Human Genetics, University of Chicago, 920 E. 58th Street, Chicago, Illinois 60637, USA. ${ }^{95}$ Department of Family and Preventive Medicine, University of California San Diego, 9500 Gilman Drive, La Jolla, California 92093, USA. ${ }^{96}$ Department of Cardiology, Division Heart and Lungs, University Medical Center Utrecht, Heidelberglaan 100, Utrecht, 3584 CX, The Netherlands. ${ }^{97}$ Durrer Center for Cardiogenetic Research, ICIN-Netherlands Heart Institute, Catharijnesingel 52, Utrecht, 3501 DG, The Netherlands. ${ }^{98}$ Institute of Cardiovascular Science, faculty of Population Health Sciences, University College London, Gower Street, London WC1E 6BT, UK. ${ }^{99}$ University of Groningen, University Medical Center Groningen, Department of Internal Medicine, Hanzeplein 1, Groningen, 9700 RB, The Netherlands. ${ }^{100}$ Department of Medicine, Columbia University, 622 W. 168th Street, New York, New York 10032, USA. ${ }^{101}$ Institute for Community Medicine, University Medicine Greifswald, W.-Rathenau-Str. 48, Greifswald 17475, Germany. ${ }^{102}$ Department of Economics, Cornell University, 480 Uris Hall, Ithaca, New York 14853, USA. ${ }^{103}$ Department of Economics and Center for Economic and Social Research, University of Southern California, 314C Dauterive Hall, 635 Downey Way, Los Angeles, California 90089, USA. ${ }^{104}$ Human Genetics Center, School of Public Health, University of Texas Health Science Center at Houston, 1200 Pressler Street, Suite 453E, Houston, Texas 77030, USA. ${ }^{105}$ Centre for Genomic and Experimenta Medicine, University of Edinburgh, Western General Hospital, Edinburgh EH4 2XU, UK. ${ }^{106}$ McKusick-Nathans Institute of Genetic Medicine, Johns Hopkins University School of Medicine, Baltimore, Maryland 21205, USA. ${ }^{107}$ Program in Genetic Epidemiology and
Statistical Genetics, Harvard School of Public Health, 665 Huntington Ave, Boston, Massachusetts 02115, USA. ${ }^{108}$ Genome Technology Branch, National Human Genome Research Institute, NIH, Bethesda, Maryland 20892, USA. ${ }^{109}$ College of Medicine, Dentistry and Nursing, Ninewells Hospital and Medical School, College Office, Level 10, Dundee DD1 9SY, UK. ${ }^{110}$ National Heart, Lung, and Blood Institute's Framingham Heart Study, 73 Mt. Wayte Ave, Framingham, Massachusetts 01702, USA. ${ }^{111}$ Psychology, University of Edinburgh, 7 George Square, Edinburgh EH8 9JZ, UK. ${ }^{112}$ Centre for Cognitive Ageing and Cognitive Epidemiology, University of Edinburgh, 7 George Square, Edinburgh EH8 9JZ, UK. ${ }^{113}$ Department of Internal Medicine B, University Medicine Greifswald, Ferdinand-Sauerbruch-Str. NK, Greifswald 17475 , Germany. ${ }^{114}$ Cardiology, Geneva University Hospitals, Rue Gabrielle-Perret-Gentil, 4, Genève 14, 1211,

Switzerland. ${ }^{115}$ Robertson Centre, University of Glasgow, Boyd Orr Building, Glasgow G12 $8 Q Q$, Scotland. ${ }^{116}$ Division of Endocrinology, Brigham and Women's Hospital and Harvard Medical School, 75 Francis St, Boston, Massachusetts 02115, USA. ${ }^{117}$ Institute of Clinical Chemistry and Laboratory Medicine, University Medicine Greifswald, FerdinandSauerbruch-Str. NK, 17475 Greifswald, Germany. ${ }^{118}$ Division of Biostatistics, Washington University, 660 S Euclid, St Louis, Missouri 63110, USA. ${ }^{119}$ Roslin Institute, University of Edinburgh, Easter Bush, Midlothian, Edinburgh EH25 9RG, UK. ${ }^{120}$ National Institutes on Aging, National Institutes of Health, Bethesda, Maryland 20892, USA. ${ }^{121}$ Department of Neurology, Boston University School of Medicine, 72 E Concord St, Boston,

Massachusetts 02118, USA. ${ }^{122}$ Department of Public Health, University of Helsinki, Hjelt Institute, P.O.Box 41, Mannerheimintie 172, Helsinki, Fl-00014, Finland.

${ }^{123}$ Musculoskeletal Research Programme, Division of Applied Medicine, University of Aberdeen, Foresterhill, Aberdeen AB25 2ZD, UK. ${ }^{124}$ State Key Laboratory of Medical Genomics, Shanghai Institute of Hematology, Rui Jin Hospital Affiliated with Shanghai Jiao Tong University School of Medicine, 197 Rui Jin Er Road, Shanghai, 200025, China. ${ }^{125}$ Department of Radiology, Erasmus Medical Center, PO Box 2040, Rotterdam, 3000 CA, The Netherlands. ${ }^{126}$ Department of Medical Sciences, Molecular Epidemiology and Science for Life Laboratory, Uppsala University, Uppsala, SE-17121, Sweden. ${ }^{127}$ Uppsala Clinical Research Center, Uppsala University, Uppsala, SE-75237, Sweden.

${ }^{128}$ Department of Chronic Disease Prevention, National Institute for Health and Welfare, P.O. Box 30, Helsinki, FI-00271, Finland. ${ }^{129}$ Department of Cardiology C5-P, Leiden University Medical Center, PO Box 9600, Leiden, 2300 RC, The Netherlands.

${ }^{130}$ Department of Clinical Physiology, University of Tampere and Tampere University Hospital, P.O. Box 2000, Tampere, Fl-33521, Finland. ${ }^{131}$ Diabetes Prevention Unit, National Institute for Health and Welfare, P.O. Box 30, Fl-00271 Helsinki, Finland. ${ }^{132}$ Department of Medicine, Division of Endocrinology, Helsinki University Central Hospital, P.O.Box 340, Haartmaninkatu 4, Helsinki, FI-00029, Finland. ${ }^{133}$ Minerva Foundation Institute for Medical Research, Biomedicum 2U, Tukholmankatu 8, Helsinki, FI-00290, Finland. ${ }^{134}$ Laboratory for Genotyping Development RCfIMS, 1-7-22 Suehirocho, Tsurumi-ku, Yokohama, Kanagawa, 230-0045, Japan. ${ }^{135}$ Department of Medicine, University of Eastern Finland and Kuopio University Hospital, Kuopio, FI-70210, Finland ${ }^{136}$ Institute of Behavioural Sciences, University of Helsinki, P.O. Box 9, University of Helsinki, Helsinki, FI-00014, Finland. ${ }^{137}$ Folkhälsan Reasearch Centre, PB 63, Helsinki, FI00014 University of Helsinki, Finland. ${ }^{138}$ Department of Clinical Chemistry, Fimlab Laboratories and School of Medicine University of Tampere, Tampere, FI-33520, Finland. ${ }^{139}$ Department of Medical Sciences, University Hospital, Uppsala, 75185, Sweden. ${ }^{140}$ Transplantation laboratory, Haartman Institute, University of Helsinki, P.O. Box 21, Helsinki, FI-00014, Finland. ${ }^{141}$ National Institute of Environmental Health Sciences, National Institutes of Health, Department of Health and Human Services, Research Triangle Park, North Carolina 27709, USA. ${ }^{142}$ Ophthalmology, Massachusetts Eye and Ear, 243 Charles Street, Boston, Massachusetts 02114, USA. ${ }^{143}$ Department of Statistics, University of Auckland, 303.325 Science Centre, Private Bag 92019, Auckland, 1142, New Zealand. ${ }^{144}$ Department of Health, Functional Capacity and Welfare, National Institute for Health and Welfare, P.O. Box 30, Helsinki, FI-00271, Finland. ${ }^{145}$ Department of Internal Medicine, University Hospital, Rue du Bugnon 44, Lausanne, 1011, Switzerland. ${ }^{146} \mathrm{Human}$ Genetics, Wellcome Trust Sanger Institute, Hinxton, Cambridge CB10 $1 \mathrm{HH}$, UK. ${ }^{147}$ Division of Allergy and Clinical Immunology, Department of Medicine, The Johns Hopkins University School of Medicine, Baltimore, Maryland 21224, USA. ${ }^{148}$ Laboratory of Molecular Medicine, Human Genome Center, Institute of Medical Science, The University of Tokyo, 4-6-1 Shirokanedai, Minato-ku, Tokyo, 108-8639, Japan. ${ }^{149}$ Division of General Internal Medicine, Massachusetts General Hospital, 50 Staniford St, Boston, Massachusetts 02114, USA ${ }^{150}$ Institute of Human Genetics, Helmholtz Zentrum München, German Research Center for Environmental Health, Ingolstädter Landstr. 1, Neuherberg 85764, Germany. ${ }^{151}$ Institute of Human Genetics, Klinikum rechts der Isar, Technische Universität München, Ismaninger Str. 22, München 81675, Germany. ${ }^{152}$ Molecular Epidemiology, QIMR Berghofer Medical Research Institute, 300 Herston Rd, Herston, Brisbane, Queensland 4006, Australia. ${ }^{153}$ Genome Science Institute, Boston University School of Medicine, 72 East Concord Street, E-304, Boston, Massachusetts 02118, USA. ${ }^{154} \mathrm{HUCH}$ Heart and Lung center, Helsinki University Central Hospital, P.O. Box 340, Helsinki, Fl-00029, Finland. ${ }^{155}$ Pulmonary Center and Department of Medicine, Boston University School of Medicine, 72 E Concord St, Boston, Massachusetts 02118, USA. ${ }^{156}$ Department of Medicine, University of Ibadan, Ibadan, Nigeria. ${ }^{157}$ ICAMS, University of Glasgow, 126 University Way, Glasgow G12 8TA, UK. ${ }^{158}$ Division of Epidemiology and Community Health, University of Minnesota, 1300 S 2nd Street, Minneapolis, Minnesota 55454, USA. ${ }^{159}$ Centre for Global Health and Department of Public Health, School of Medicine, University of Split, Soltanska 2,21000 Split, Croatia. ${ }^{160}$ Obesity Research Unit, Research Programs Unit, Diabetes and Obesity, University of Helsinki, P.O.Box 63, Haartmaninkatu 8, FI-00014, Helsinki, Finland. ${ }^{161}$ International Centre for Circulatory Health, Imperial College London, London W2 1LA, UK.

${ }^{162}$ Department of Genomics of Common Disease, School of Public Health, Imperial College London, London SW7 2AZ, UK. ${ }^{163}$ Department of Cardiology and Cardio thoracic Surgery Hero DMC Heart Institute, Civil Lines, 141001, Ludhiana, India. ${ }^{164}$ Department Public Health Sciences, University of Virginia School of Medicine, 3232 West Complex, Charlottesville, Virginia 22908, USA. ${ }^{165}$ Department of Psychological \& Brain Sciences, Indiana University Bloomington, 1101 E. 10th Street, Bloomington, Indiana 47405, USA. ${ }^{166}$ Institute of Molecular Biology and Biochemistry, Medical University Graz, 
Harrachgasse 21, Graz, A-8010, Austria. ${ }^{167}$ Science for Life Laboratory, Karolinska Institutet, Stockholm, SE-17121, Sweden. ${ }^{168}$ University of Dundee, Kirsty Semple Way, Dundee DD2 4DB, UK. ${ }^{169}$ Cardiovascular Health Research Unit, Division of Cardiology, University of Washington, 1730 Minor Ave, Suite 1360, Seattle, Washington 98101, USA. ${ }_{170}$ Molecular and Cellular Therapeutics, Royal College of Surgeons in Ireland, St. Stephen's Green, Dublin 2, Ireland. ${ }^{71}$ UMR INSERM U1122; IGE-PCV “Interactions Gène-Environnement en Physiopathologie Cardio-Vasculaire", INSERM, University of Lorraine, 30 Rue Lionnois, 54000 Nancy, France. ${ }^{172}$ Institute of Medical Informatics, Biometry and Epidemiology, Chair of Genetic Epidemiology, Ludwig-MaximiliansUniversität, Munich 81377, Germany. ${ }^{173}$ Department of Endocrinology, All India Institute of Medical Sciences, Ansari Nagar East, New Delhi, 110029, India. ${ }^{174}$ National Heart and Lung Institute, Imperial College London, Du Cane Road, London W12 ONN, UK. ${ }^{175}$ Department of Public Health Sciences. Stritch School of Medicine, Loyola University Chicago, Maywood, Illinois 60153, USA. ${ }^{176}$ NIHR Leicester Cardiovascular Biomedical Research Unit, University of Leicester, Glenfield Hospital, Groby Road, Leicester LE3 9QP, UK. ${ }^{177}$ Institute of Molecular and Cell Biology, University of Tartu, Riia 23, Tartu, 51010, Estonia. ${ }^{178}$ Centre for Vascular Prevention, Danube-University Krems, $3500 \mathrm{Krems}$, Austria. ${ }^{179}$ Diabetes Research Group, King Abdulaziz University, 21589 Jeddah, Saudi Arabia. ${ }^{180}$ Department of Internal Medicine, Erasmus Medical Center, PO Box 2040, Rotterdam, 3000 CA , The Netherlands. ${ }^{181}$ Department of Epidemiology, Johns Hopkins Bloomberg School of Public Health, Baltimore, Maryland 21205, USA. ${ }^{82}$ Finnish Lung Health Association, Sibeliuksenkatu 11 A 1, Helsinki, Fl-00250, Finland. ${ }^{183}$ Genetic Epidemiology, QIMR Berghofer Medical Research Institute, 300 Herston Rd, Herston, Brisbane, Queensland 4006, Australia. ${ }^{184}$ Diabetes Unit, KEM Hospital and Research Centre, Rasta Peth, Pune, 411011, India. ${ }^{185}$ Renal Unit, Department of Medicine, University of Verona, Piazzale A. Stefani 1, 37124 Verona, Italy. ${ }^{186}$ Department of Epidemiology and Biostatistics, Case Western Reserve University, Cleveland, Ohio 44106 USA. ${ }^{187}$ Department of Medicine, Stanford University, 300 Pasteur Drive, Stanford, California 94305, USA. ${ }^{188}$ Department of Pediatrics, Perelman School of Medicine, The University of Pennsylvania, 3615 Civic Center Boulevard, Philadelphia, Pennsylvania 19104, USA. ${ }^{189}$ Translational Gerontology Branch, National institute on Aging, Baltimore, Maryland 21225, USA. ${ }^{190}$ Usher Institute for Population Health Sciences and Informatics, University of Edinburgh, No. 9 Edinburgh Bioquarter, 9 Little France Road, Edinburgh EH16 4UX, UK. ${ }^{191}$ Centre for Pharmacogenetics and Pharmacogenomics, Medical Research Institute, University of Dundee, Ninewells Hospital and School of Medicine, Dundee DD1 9SY, UK. ${ }^{192}$ Princess Al-Jawhara Al-Brahim Centre of Excellence in Research of Hereditary Disorders (PACER-HD), King Abdulaziz University, Jeddah, 21589, Saudi Arabia. ${ }^{193}$ Faculty of Medicine, Imperial College London, Charing Cross Campus, St Dunstan's Road, London W6 8RP, UK. ${ }^{194}$ Department of Medicine, University of Leipzig, Leipzig 04103, Germany. ${ }^{195}$ Institute of Preventive Medicine, Bispebjerg and Frederiksberg Hospital, The Capital Region, Copenhagen, 2000, Denmark.

${ }^{196}$ Department of Epidemiology, University of Alabama at Birmingham, 1665 University Boulevard, Birmingham, Alabama 35294, USA. ${ }^{197}$ Department of Psychiatry, University Medical Center Groningen, University of Groningen, P.O. Box 30.001, Groningen, 9700 $\mathrm{RB}$, The Netherlands. ${ }^{198}$ Epidemiology of diabetes, obesity and chronic kidney disease over the lifecourse, Inserm, CESP Center for Research in Epidemiology and Population Health U1018, 16 Avenue Paul Vaillant Couturier, 94807 Villejuif, France.

${ }^{199}$ Dipartimento di Scienze Mediche, Catholic University of the Sacred Heart, Via G. Moscati 31/34, 00168 Roma, Italy. ${ }^{200}$ Department of Biostatistics, University of Liverpool, Duncan Building, Daulby Stree, Liverpool L69 3GA, UK. ${ }^{201}$ Department of General Practice and Primary Health Care, University of Helsinki, P.O. Box 20, University of Helsinki, Helsinki, Fl-00014, Finland. ${ }^{202}$ Vasa Central Hospital, Sandviksgatan 2-4, Vasa, Fl-65130, Finland. ${ }^{203}$ Folkhälsan Reasearch Centre, PB 63, University of Helsinki,
Helsinki, Fl-00014, Finland. ${ }^{204}$ Unit of General Practice, Helsinki University Central Hospital, Haartmaninkatu 4, Helsinki, Fl-00290, Finland. ${ }^{205}$ Neuro-Imaging Genetics, QIMR Berghofer Medical Research Institute, 300 Herston Rd, Herston, Brisbane,

Queensland 4006, Australia. ${ }^{206}$ Cardiovascular Genetics Division, University of Utah, 420 Chipeta Way, Room 1160, Salt Lake City, Utah 84117, USA. ${ }^{207}$ Alzheimer Scotland Research Centre, University of Edinburgh, 7 George Square, Edinburgh EH8 9JZ, UK. ${ }^{208}$ Department of Psychiatry, Erasmus Medical Center, PO Box 2040, Rotterdam, 3000 CA, The Netherlands. ${ }^{209}$ National Institute for Health and Welfare (THL), P.O.Box 30, Mannerheimintie 166, Helsinki, Fl-00271, Finland. ${ }^{210}$ Department of Kinesiology, Laval University, 2300 rue de la Terrasse, Quebec G1V 0A6, Canada. ${ }^{211}$ Department of Physiology and Biophysics, University of Mississippi Medical Center, 2500 N. State Street Jackson, Mississippi 39216, USA. ${ }^{212}$ Department of Clinical Physiology and Nuclear Medicine, University of Turku and Turku University Hospital, Turku, Fl-20521, Finland. ${ }^{213}$ Research Center of Applied and Preventive Cardiovascular medicine, University of Turku, Turku, Fl-20521, Finland. ${ }^{214}$ University of Groningen, University Medical Center Groningen, Department of Genetics, Hanzeplein 1, Groningen, 9700 RB, The Netherlands. ${ }^{215}$ Department of Economics, Stockholm School of Economics, Box 6501 , Stockholm, SE113 83, Sweden. ${ }^{216}$ Department of Medical Epidemiology and Biostatistics, Karolinska Institutet, Box 281, Stockholm, SE-171 77, Sweden. ${ }^{217}$ Department of Genetics and Biostatistics, Washington University School of Medicine, 4444 Forest Park Boulevard, Saint Louis, Missouri 63108, USA. ${ }^{218}$ Department of Health Policy and Management, Johns Hopkins Bloomberg School of Public Health, Baltimore, Maryland 21205, USA ${ }^{219}$ Department of Pediatrics, University of Oklahoma Health Sciences Center, 940 Stanton Young Boulevard, Oklahoma City, Oklahoma 73104, USA. ${ }^{220}$ Department of Pharmaceutical Sciences, University of Oklahoma Health Sciences Center, Oklahoma City, Oklahoma 73104, USA. ${ }^{221}$ Sidra Medical and Research Centre, Doha, Qatar. ${ }^{222}$ The Mindich Child Health and Development Institute, Icahn School of Medicine at Mount Sinai, One Gustave L. Levy Place, New York, New York 10029, USA. ${ }^{223}$ Genome Institute of Singapore, 60 Biopolis Street, \#02-01 Genome, Singapore, 138672, Singapore. ${ }^{224}$ Program for Personalised and Genomic Medicine, Department of Medicine, University of Maryland School of Medicine, 685 Baltimore St. MSTF, Baltimore, Maryland 21201, USA. ${ }^{225}$ Geriatric Research and Education Clinical Center, Veterans Administration Medical Center, 685 W Baltimore MSTF, Baltimore, Maryland 21201, USA. ${ }^{226}$ BHF Glasgow Cardiovascular Research Centre, University of Glasgow, 126 University Place, Glasgow G12 8TA, UK. ${ }^{227}$ Carolina Center for Genome Sciences, University of North Carolina at Chapel Hill, 137 E. Franklin Street, Suite 306, Chapel Hill, North Carolina 27599, USA. ${ }^{228}$ Cardiovascular Health Research Unit, Departments of Medicine, Epidemiology and Health Services, University of Washington, 1730 Minor Ave, Suite 1360, Seattle, Washington 98101, USA. ${ }^{229}$ Group Health Research Institute, Group Health Cooperative, 1730 Minor Ave, Suite 1360, Seattle, Washington 98101, USA. ${ }^{230}$ Imperial College Healthcare NHS Trust, Imperial College London, Praed Street, London W2 $1 \mathrm{NY}$, UK. ${ }^{231}$ Population Health Research Institute, St George's, University of London, Cranmer Terrace, London SW17 ORE, UK. ${ }^{232}$ Steno Diabetes Centre, Niels Steensens Ve 2, Gentofte, 2820, Denmark. ${ }^{23}$ Translational Laboratory in Genetic Medicine (TLGM), Agency for Science, Technology and Research (A*STAR), 8A Biomedical Grove, 138648, Singapore. ${ }^{234}$ Centre for Population Health Sciences, Usher Institute for Population Health Sciences and Informatics, University of Edinburgh, Teviot Place, Edinburgh EH8 9AG, UK. ${ }^{235}$ Institutes for Neurogenetics and Integrative \& Experimental Genomics, University of Lübeck, Lübeck 23562, Germany. ${ }^{236}$ School of Biotechnology, Jawaharlal Nehru University, New Delhi 110067, India.

*These authors contributed equally to this work. 


\section{RESEARCH LETTER}

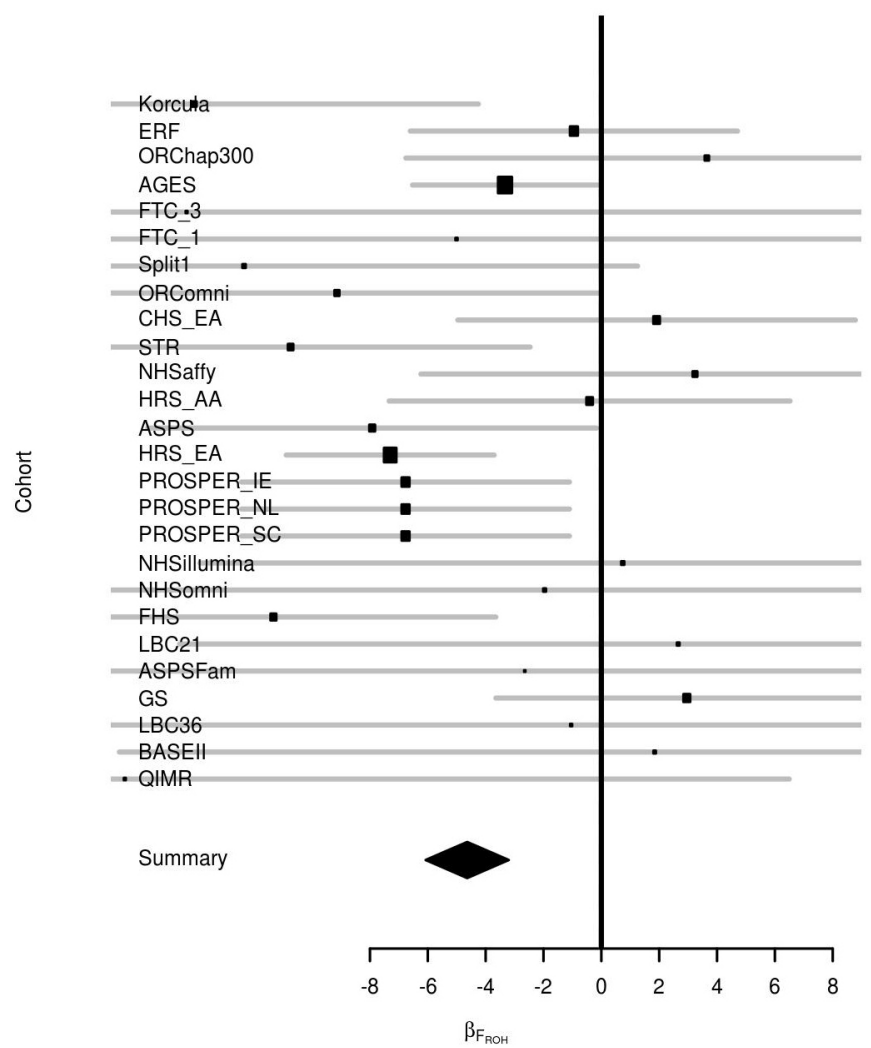

Extended Data Figure $1 \mid$ Forest plot for cognitive ability (g). Individual sub-cohort estimates of effect size and the $95 \%$ confidence interval are plotted. Sub-cohorts are ordered from top to bottom according to their weight in the meta-analysis, so larger or more homozygous cohorts appear towards the top. The scale of $\beta_{F_{\mathrm{ROH}}}$ is in intra-sex standard deviations. The meta-analytical estimate is displayed at the bottom. Sub-cohort names follow the conventions detailed in Supplementary Table 6 and the Supplementary Table 11 legend. Sample sizes, effect sizes and $P$ values for association are given in Table 1 . This trait was rank-transformed. 


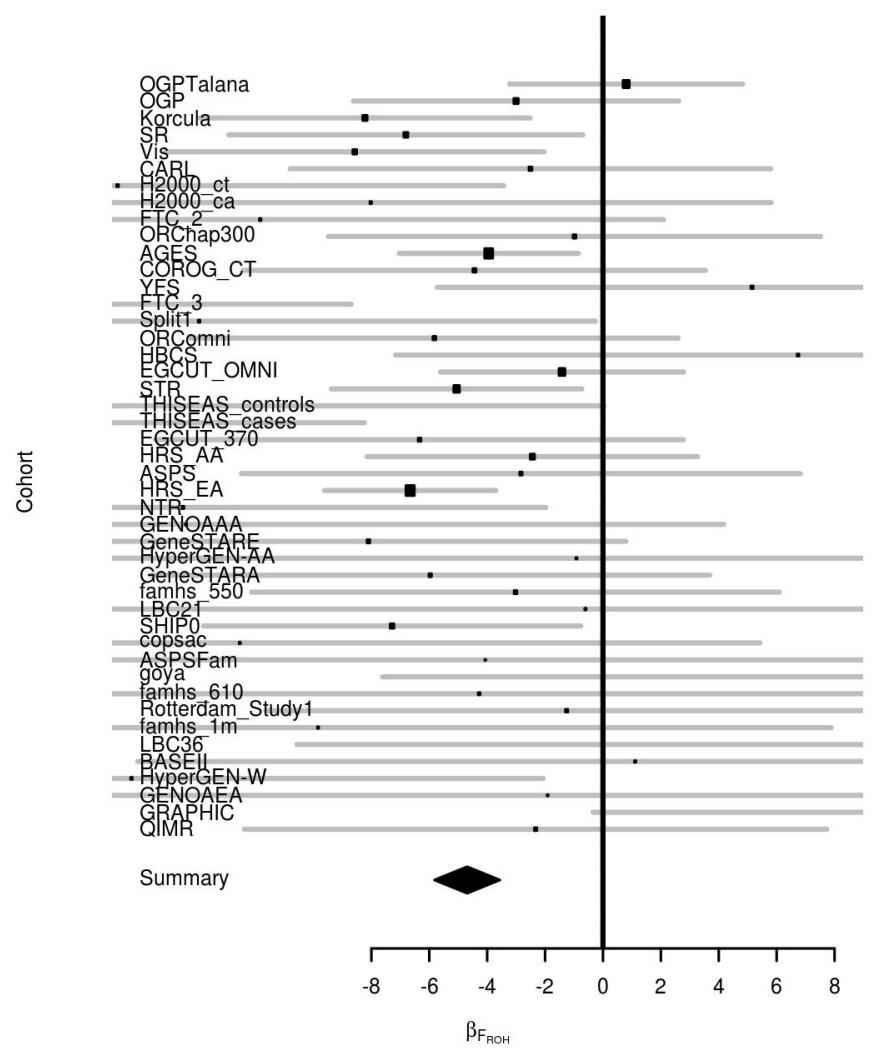

Extended Data Figure $2 \mid$ Forest plot for educational attainment. Individual sub-cohort estimates of effect size and the $95 \%$ confidence interval are plotted. Sub-cohorts are ordered from top to bottom according to their weight in the meta-analysis, so larger or more homozygous cohorts appear towards the top. The scale of $\beta_{F_{\mathrm{p}} \text { is }}$ in intra-sex standard deviations. The meta-analytical estimate is displayed at the bottom. Sub-cohort names follow the conventions detailed in Supplementary Table 6 and the Supplementary Table 11 legend. Sample sizes, effect sizes and $P$ values for association are given in Table 1. 


\section{RESEARCH LETTER}

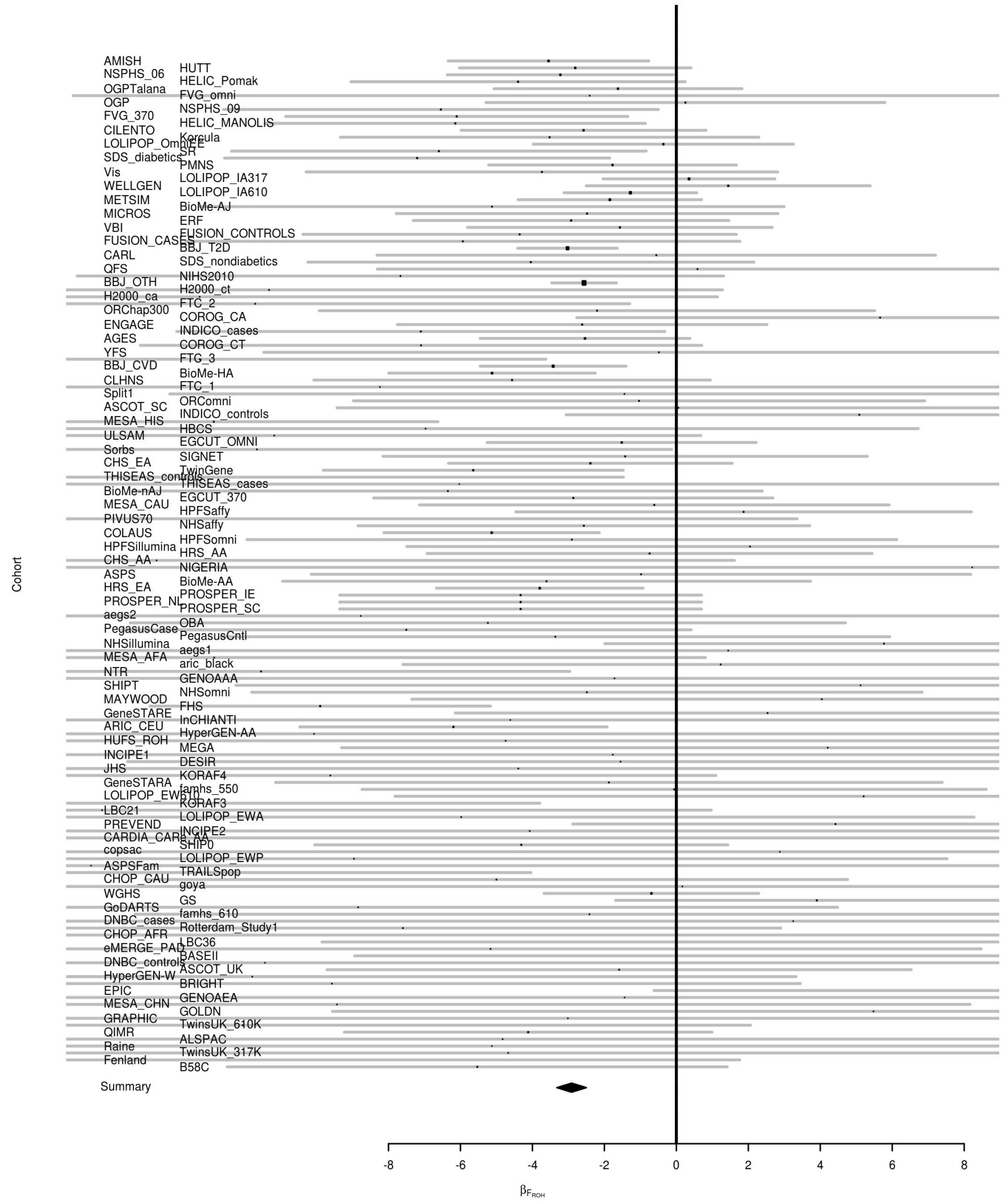

Extended Data Figure 3 Forest plot for height. Individual sub-cohort estimates of effect size and the $95 \%$ confidence interval are plotted. Sub-cohorts are ordered from top to bottom according to their weight in the meta-analysis, so larger or more homozygous cohorts appear towards the top. The scale of
$\beta_{F_{\mathrm{ROH}}}$ is in intra-sex standard deviations. The meta-analytical estimate is displayed at the bottom. Sub-cohort names follow the conventions detailed in Supplementary Table 6 and the Supplementary Table 11 legend. Sample sizes, effect sizes and $P$ values for association are given in Table 1 . 


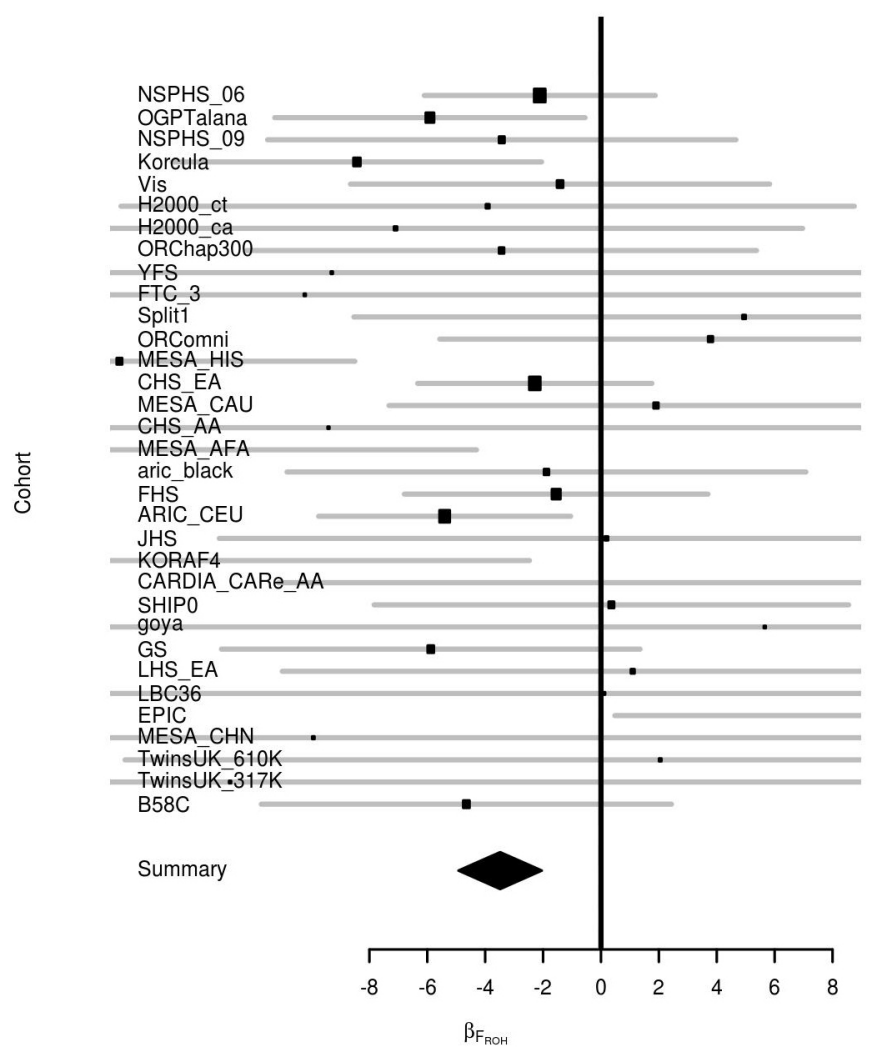

Extended Data Figure $4 \mid$ Forest plot for forced expiratory lung volume in one second. Individual sub-cohort estimates of effect size and the $95 \%$ confidence interval are plotted. Sub-cohorts are ordered from top to bottom according to their weight in the meta-analysis, so larger or more homozygous cohorts appear towards the top. The scale of $\beta_{F_{\mathrm{RO}}}$ is in intra-sex standard deviations. The meta-analytical estimate is displayed at the bottom. Sub-cohort names follow the conventions detailed in Supplementary Table 6 and the Supplementary Table 11 legend. Sample sizes, effect sizes and $P$ values for association are given in Table 1. This trait was rank-transformed. 
Cognitive g+

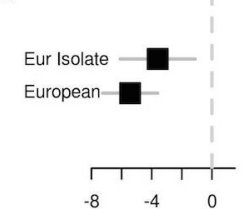

b

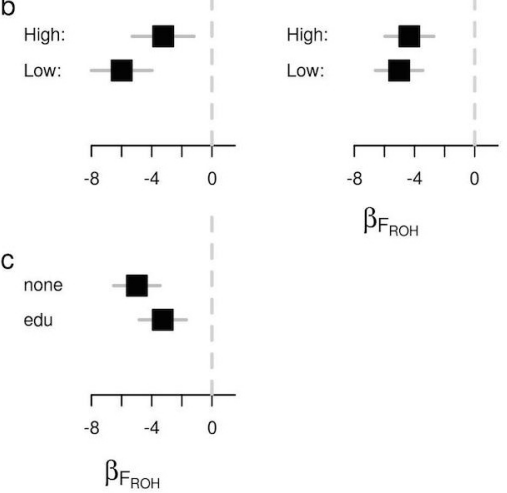

Extended Data Figure $5 \mid$ Signals of directional dominance are robust to stratification by geography or demographic history or inclusion of educational attainment as covariate. a, Cohorts are divided by continental biogeographic ancestry (African (15 sub-cohorts), East Asian (5), South and Central Asian (SC Asian; 10), Hispanic (3)), with Europeans being divided into Finns (13), other European isolates (self-declared, 23), and (non-isolated) Europeans (90). Meta-analysis was carried out for all subsets with 2,000 or more samples available. Sample numbers are as follows: cognitive $g$, Eur isolate, 6,638 ; European, 44,153; educational attainment, African 4,811; Eur isolate, 8,032; European, 55,549; Finland 9,068; height, African, 21,500; E Asian, 30,011; Eur isolate, 23,116; European, 228,813, Finland, 30,427, Hispanic, 5,469, SC Asian, 13,523; FEV1, African, 6,604, Eur isolate, 4,837, European, 49,223, Finland, 2,340 . $\beta_{F_{\mathrm{ROH}}}$ is consistent across geography and in both isolates and more cosmopolitan populations. b, Cohorts were divided into high and low $\mathrm{ROH}$ strata of equal power and meta-analysis repeated - the effects are consistent
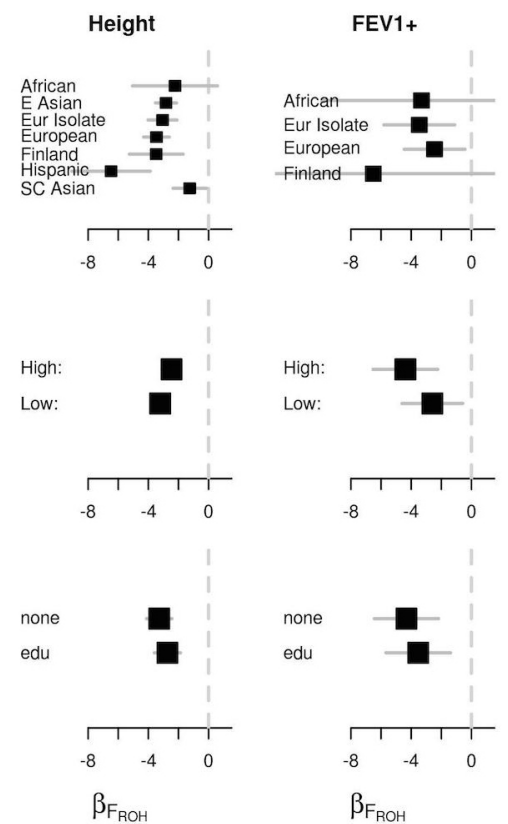

across strata for all four traits. The mean SROH for the high and low strata, respectively, are 13.4 and $4.3 \mathrm{Mb}$ for cognitive $g$; 28.1 and 5.1 $\mathrm{Mb}$ for educational attainment; 31.9 and $10.8 \mathrm{Mb}$ for height; and 41.4 and $4.5 \mathrm{Mb}$ for FEV1. c, To assess the potential for socio-economic confounding, where available, educational attainment was included in the regression model (edu) and compared to a model without educational attainment (none) in the same subset of cohorts. The signals reduce slightly when the education covariate is included; the analysis is not possible for educational attainment as a trait. For cognitive $g$, numbers of subjects are 36,847 and 36,023; for height 131,614 and 120,945; and for FEV1, 15,717 and 15,425, for edu and none, respectively. The numbers differ because of missing individual educational data within cohorts. Plus signs indicate that the phenotype was rank-transformed. Trait units are intra-sex standard deviations and the genomic measure is unpruned SROH. Subset estimates of effect size for FROH and the 95\% confidence are plotted. 

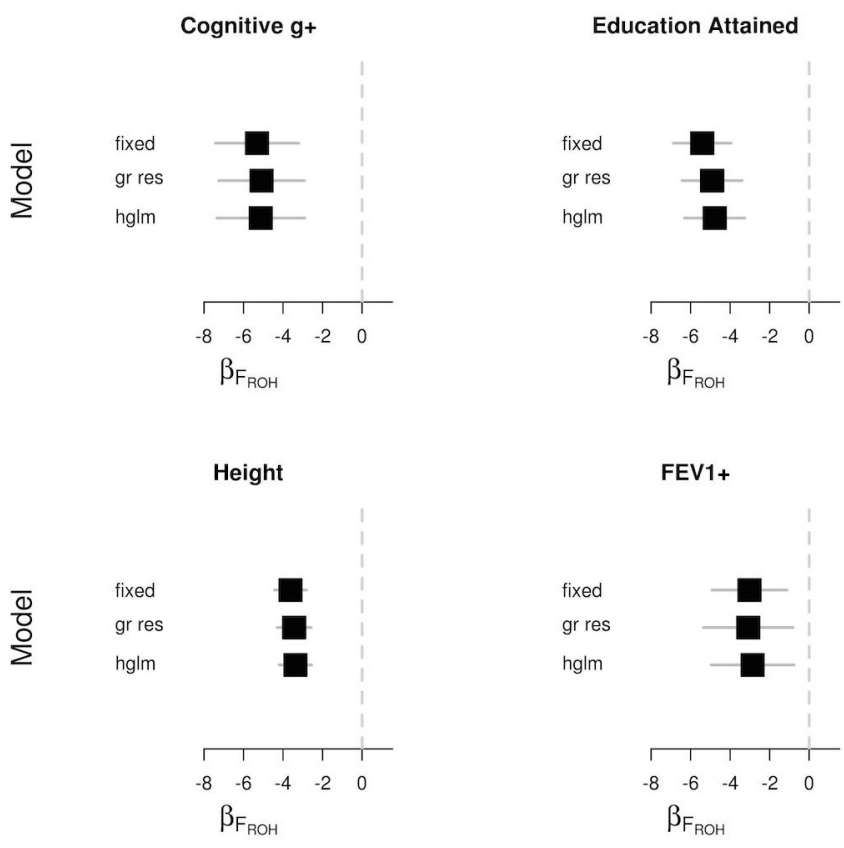

Extended Data Figure 6 Signals of directional dominance are robust to model choice. Meta-analytical estimates of effect size and standard errors are plotted for various models. Fixed, no mixed modelling was used; gr res,

GRAMMAR + residuals were fitted; hglm, full hierarchical generalized linear mixed model was used. Plus signs indicate that the phenotype was rank-

transformed. 15,355 subjects were used for cognitive $g, 36,060$ for educational attainment, 89,112 for height and 15,262 for FEV1. 


\section{RESEARCH LETTER}
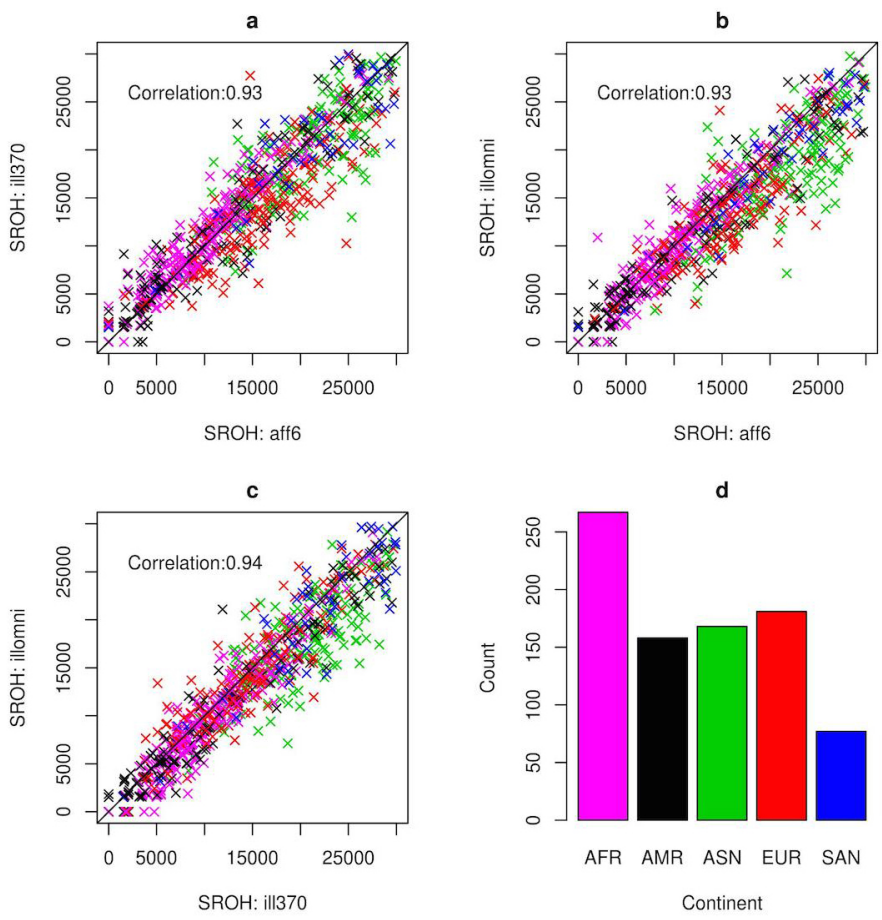

Extended Data Figure $7 \mid$ Correlation in SROH for different genotyping arrays using HapMap populations. a-c, $x$ and $y$ axes show $\mathrm{SROH}$ from 0-30 Mb. ill370, Illumina CNV370; aff6, Affymetrix6; illomni, Illumina OmniExpress. The graphs are shown for the specific PLINK call parameters used. d, Sample numbers per continent are presented in a bar chart. AFR, African; AMR, mixed American; ASN, East Asian; EUR, European; SAN, South Asian. Only samples with SROH below $30 \mathrm{Mb}$ are plotted, to be conservative to the effect of outliers, which have very strongly correlated estimates of $\mathrm{SROH}$ ( $r=0.96-0.97$ for comparisons including such very homozygous individuals). In these plots, the correlation between $\mathrm{SROH}$ called by the two arrays, $r=0.93-0.94$. 
Extended Data Table 1 | Continental ancestry of cohorts participating in each trait study.

\begin{tabular}{|l|r|r|r|r|r|r|r|}
\hline & African & East Asian & European & Hispanic & \multicolumn{2}{l}{ S/C Asian } & All \\
\hline BMI & $21689 / 15$ & $29009 / 5$ & $279400 / 117$ & $7836 / 3$ & $13464 / 10$ & $351398 / 150$ \\
\hline Cognitive $g$ & $1539 / 1$ & NA/NA & $49559 / 22$ & - & - & $51098 / 23$ \\
\hline Diastolic BP & $17074 / 12$ & $24200 / 5$ & $204742 / 85$ & $7284 / 3$ & $12876 / 9$ & $266176 / 114$ \\
\hline Education Attained & $4811 / 4$ & NA/NA & $79576 / 42$ & - & $338 / 1$ & $84725 / 47$ \\
\hline Fasting Insulin & $6895 / 8$ & $1603 / 1$ & $72006 / 49$ & - & $6303 / 5$ & $86807 / 63$ \\
\hline FEV1 & $6604 / 5$ & $617 / 1$ & $58089 / 27$ & $825 / 1$ & - & $66135 / 34$ \\
\hline FEV1/FVC & $6565 / 5$ & $616 / 1$ & $57888 / 27$ & $822 / 1$ & - & $65891 / 34$ \\
\hline FP Glucose & $8942 / 9$ & $1615 / 1$ & $122368 / 74$ & $1938 / 1$ & $6921 / 5$ & $141784 / 90$ \\
\hline HbA1C & $6629 / 4$ & $694 / 1$ & $92732 / 31$ & $4038 / 2$ & $7509 / 4$ & $111602 / 42$ \\
\hline HDL Cholesterol & $15099 / 13$ & $10478 / 5$ & $215621 / 92$ & $4426 / 3$ & $12508 / 9$ & $258132 / 122$ \\
\hline Height & $20300 / 14$ & $30011 / 5$ & $281369 / 114$ & $5469 / 2$ & $13523 / 10$ & $350672 / 145$ \\
\hline LDL Cholesterol & $13375 / 11$ & $2503 / 2$ & $172245 / 77$ & $4340 / 3$ & $11186 / 8$ & $203649 / 101$ \\
\hline Systolic BP & $17023 / 12$ & $24424 / 5$ & $205253 / 85$ & $7225 / 3$ & $12859 / 9$ & $266784 / 114$ \\
\hline Total Cholesterol & $15130 / 13$ & $20187 / 5$ & $209421 / 91$ & $4491 / 3$ & $11674 / 8$ & $260903 / 120$ \\
\hline Triglycerides & $13886 / 12$ & $2542 / 2$ & $181526 / 84$ & $2745 / 2$ & $10688 / 7$ & $211387 / 107$ \\
\hline Waist-hip ratio & $8182 / 7$ & $2549 / 2$ & $171753 / 73$ & $1446 / 1$ & $12598 / 9$ & $196528 / 92$ \\
\hline
\end{tabular}

The first number in each cell is the number of participants with that continental ancestry. The second number is the number of sub-cohorts. S/C Asian, South and Central Asian. 